\title{
红树来源青霉属真菌次级代谢产物与生物活性(2007～2020)
}

\author{
曾尾女 黄国雷王斌蔡瑾郑彩娟* \\ (海南师范大学化学与化工学院 热带药用资源化学教育部重点实验室 \\ 海南省热带药用植物化学重点实验室 海口 571158)
}

\begin{abstract}
摘要 红树植物生长于缺氧、酸性、含盐的热带和亚热带地区的潮间带, 生长环境的特殊性为红树来源真菌提供了特 殊的宿主环境. 红树来源真菌独特的遗传背景和代谢途径使得其次级代谢产物具有结构新颖且类型多样、生物活性多 样化等特点. 青霉属(Penicillium sp.)真菌分布广泛, 为红树来源真菌的一大类群, 能够代谢产生生物碱类、肽类、萜类 及聚酮类等结构类型多样且新颖的代谢产物，大多具有抗肿瘤、抗炎、抗菌和酶抑制剂等多种生物活性. 综述了 2007 2020 年, 从红树来源青需属真菌中发现的 276 个新化合物, 介绍了其结构和生物活性, 以期为后续红树来源青霉属真 菌新型天然药物的开发提供参考.
\end{abstract}

关键词 红树; 青霉属真菌; 次级代谢产物; 生物活性

\section{Secondary Metabolites and Bioactivities of Penicillium sp. Sourced from Mangrove from 2007 to 2020}

\author{
Zeng, Weinü Huang, Guolei Wang, Bin Cai, Jin Zheng, Caijuan* \\ (Key Laboratory of Tropical Medicinal Plant Chemistry of Hainan Province, Key Laboratory of Tropical Medicinal \\ Resources Chemistry of Ministry of Education, College of Chemistry and Chemical Engineering, \\ Hainan Normal University, Haikou 571158)
}

\begin{abstract}
Mangrove plants grow in tropical and subtropical intertidal zones with acid, salt and oxygen deficit. The particularity of the growth environment provides a special host environment for mangrove fungi. The unique genetic background and metabolic pathway of mangrove-derived fungi make their secondary metabolites have novel structures and diverse biological activities. The genus of Penicillium is widely distributed, which is a large group of mangrove-derived fungi. It can produce metabolites with various structural types and novel structures such as alkaloids, peptides, terpenes, polyketones and other metabolites. Most of them have anti-tumor, anti-inflammatory, anti-bacterial, enzyme inhibitors and other bioactivities. The advances of the 276 natural products produced from mangrove-derived Penicillium in recent 14 years (from 2007 to 2020) are reviewed, in order to provide reference for the development of new natural drugs of mangrove Penicillium sp. fungi.

Keywords mangrove; Penicillium sp.; secondary metabolite; bioactivity
\end{abstract}

红树林是生长分布在热带和亚热带地区的潮间带 河口地带的植物群落, 其生境具有缺氧、酸性及含盐等 特征 ${ }^{[1-2]}$. 其所处的环境特殊, 不仅使红树植物演生出特 殊的次级代谢产物生物合成途径, 而且为红树来源真菌 提供了特殊的宿主环境, 使得红树来源真菌能够代谢产 生结构新颖、活性显著的次级代谢产物, 从而成为海洋 药物先导化合物发现的重要源泉. 本文总结了近 14 年 (2007 2020 年)红树来源青霉属真菌资源研究的概况,
分析了红树来源青霉属真菌代谢产物的结构与生物活 性, 以期为红树来源青霉属真菌的进一步研究提供合理 可靠的理论依据.

\section{1 红树来源青霉属真菌多样性研究}

青霉属(Penicillium)真菌是广泛存在于自然环境中 的一类腐生类真菌，也是红树林植物中分布较广泛的一 个属. 其和大部分腐生类真菌一样可以产生丰富多样的

\footnotetext{
* Corresponding author. E-mail: caijuan2002@163.com

Received March 24, 2021; revised June 14, 2021; published online July 12, 2021.

Project supported by the National Natural Science Foundation of China (No. 31760093), the Natural Science Foundation of Hainan Province (Nos. 220RC593, 218MS045) and the Key Research and Development Program of Hainan Province (No. ZDYF2019183).

国家自然科学基金(No. 31760093)、海南省自然科学基金(Nos. 220RC593, 218MS045)及海南省重点科技计划(No. ZDYF2019183)资助项目.
} 
次级代谢产物, 这些次级代谢产物大多具有丰富的生物 活性. 近 14年(2007 2020年)来关于红树植物来源真菌 的研究越来越多, 其中对青霉属真菌研究也较为广泛. 据文献统计表明, 已从秋茄 (Kandelia candel)、木榄 (Bruguiear gymnorrhiza)、尖瓣海莲(Bruguiera Sexangula var. rhynchopetala)、老鼠簕(Acanthus ilicifolius)、黄槿 (Hibiscus tiliaceus)、红海榄(Rhizophora stylosa)、白骨壤 (Avicennia marina) 等 18 种红树植物分离出的 57 株青霉 属真菌进行次级代谢产物研究(表 1) ${ }^{[1-87]}$. 其中对青霉属 真菌 Penicillium citrinum、Penicillium janthinellum、
Penicillium commune、Penicillium brocae 的代谢产物研 究的较为广泛. 例如从桔青霉 $(P$. citrinum $)$ 中分离得到 了 13 个代谢产物, 这些代谢产物具有抗菌及细胞毒活

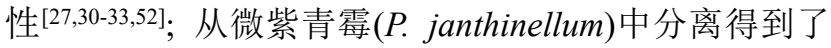
28 个次级代谢产物, 这些代谢产物具有抗菌和细胞毒 活性 ${ }^{[4-7,64-67]}$; 从 P. brocae 中分离得到了 27 个次级代谢 产物, 这些代谢产物具有抗菌、抗氧化及细胞毒活 性 [43-46,48-49]; 从团青霉( $P$. commune) 中分离得到了 10 个 异香豆素类次级代谢产物，均对 $\alpha$-葡萄糖苷酶具有较强 的抑制作用 ${ }^{[20]}$.

表 1 红树来源青霉属内生真菌分布 ${ }^{a}$

Tabel 1 Distribution of Endophytic Fungi of the Penicillium sp. from Mangrove

\begin{tabular}{|c|c|c|c|}
\hline Plant species & Isolated fungi & Site & Ref. \\
\hline \multirow{11}{*}{ Rhizospheric soil of mangrove plants } & Penicillium javanicum HK1-22 & 海南东寨港自然保护区 & [1] \\
\hline & Penicillium sp. HK1-22 & 海南东寨港自然保护区 & {$[3]$} \\
\hline & Penicillium janthinellum HK1-6 & 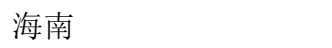 & [4-7] \\
\hline & Penicillium sp. SYFz-1 & 海南三亚 & {$[8-9]$} \\
\hline & Penicillium sp. SCSIO041218 & 三亚 & {$[10,11]$} \\
\hline & Penicillium pinophilum SCAU037 & 中国特呈岛 & [12] \\
\hline & Penicillium pinophilum $\mathrm{H} 608$ & 厦门 & [13] \\
\hline & Penicillium camemberti OUCMDZ-1492 & 海南文昌 & [14-16] \\
\hline & Penicillium herquei MA-370 & 海南 & [17] \\
\hline & Penicillium polonicum $\mathrm{H} 175$ & 福建张江口 & {$[18]$} \\
\hline & Penicillium polonicum MCCC3A00951 & 福建张江口 & [19] \\
\hline \multirow{7}{*}{ Kandelia candel } & Penicillium commune QQF-3 & 广东 & {$[20]$} \\
\hline & Penicillium aculeatum No. 9EB & 广东阳江 & {$[21]$} \\
\hline & Penicillium chermesinum ZH4-E2 & 广东 & {$[22]$} \\
\hline & Penicillium sp. sk5GW1L & 广西 & [23-24] \\
\hline & Penicillium sp. sk14JW2P & 广西 & [25] \\
\hline & Penicillium sp. (SBE-8) & 香港 & {$[26]$} \\
\hline & Penicillium citrinum QJF-22 & 海南海口 & {$[27]$} \\
\hline \multirow{4}{*}{ Bruguiera sexangula var. rhynchopetala } & Penicillium sp. TGM112 & 海南东寨港自然保护区 & [28-29] \\
\hline & Penicillium citrinum HL-5126 & 海南东寨港自然保护区 & [30-33] \\
\hline & Penicillium simplicissimum MA-332 & 海南 ～～～～～～～～～ & [34-35] \\
\hline & Penicillium sp. (J41221) & 海南东寨港自然保护区 & [36] \\
\hline \multirow{3}{*}{ Bruguiear gymnorrhiza } & Penicillium sp. GD6 & 广州湛江 & [37-39] \\
\hline & Penicillium thomi & 广西 & [40] \\
\hline & Penicillium sp. MA-37 & 海南 & [41-42] \\
\hline \multirow{5}{*}{ Avicennia marina } & Penicillium brocae MA-231 & 海南 & [43-47] \\
\hline & Penicillium sp. FJ-1 & 福建 & [48] \\
\hline & Penicillium brocae MA-192 & 海南 & [49-50] \\
\hline & Penicillium sp. ZH16 & 海南东寨港自然保护区 & [51] \\
\hline & Penicillium citrinum & 福建 & [52] \\
\hline \multirow{5}{*}{ Acanthus ilicifolius } & Penicillium chrysogenum HND11-24 & 海南 & [53] \\
\hline & Penicillium dipodomyicola HN4-3A & 海南 & {$[54]$} \\
\hline & Penicillium sp. (ZZF 32\#) & 广东湛江 & {$[55]$} \\
\hline & Penicillium sp. 299\# & 海南 & {$[56]$} \\
\hline & Penicillium sp. FJ-1 & 海南 & [57] \\
\hline \multirow{2}{*}{ Rhizophora stylosa } & Penicillium oxalicum EN-201 & 海南 & [58] \\
\hline & Penicillium solitum GWQ-143 & 1 & [59] \\
\hline Cerbera manghas & Penicillium sp. HN29-3B1 & 海南东寨港自然保护区 & [60-61] \\
\hline
\end{tabular}




\begin{tabular}{|c|c|c|c|}
\hline Plant species & Isolated fungi & Site & Ref. \\
\hline \multirow{2}{*}{ Aegiceras corniculatum } & Penicillium sp. JP-1 & 福建 & {$[62]$} \\
\hline & Penicillium sp. (strain HKI0459) & 福建厦门 & {$[63]$} \\
\hline Sonneratia caseolaris & Penicillium janthinellum HDN13-309 & 海南 & [64-67] \\
\hline \multirow{2}{*}{ Ceriouiopps tagal } & Penicillium sp. J-54 & 海南东寨港自然保护区 & {$[2,68]$} \\
\hline & Penicillium sp. JY246 & 南海 & [69] \\
\hline \multirow{2}{*}{ Lumnitzera racemosa } & Penicillium sumatrense MA-92 & 海南文昌 & {$[70]$} \\
\hline & Penicillium bilaiae MA-267 & 海南 & [71-72] \\
\hline Myoporum bontioides & Penicillium chrysogenum V11 & 中国雷州半岛 & [73-75] \\
\hline Laguncularia racemose & Penicillium coffeae MA-314 & 海南岛 & [76] \\
\hline Sonneratia apetala & Penicillium sp. ZJ-SY2 & 广东 & {$[77]$} \\
\hline \multirow{2}{*}{ Hibiscus tiliaceus } & Penicillium aurantiogriseum $328^{\#}$ & 广东 & [78] \\
\hline & Penicillium commune G2M & 海南 & [79] \\
\hline \multirow{2}{*}{ Heritiera littoralis } & Penicillium chermesinum strain HLit-ROR2 & 泰国 & {$[80]$} \\
\hline & Penicillium sp. YYSJ-3 & 广东珠海 & [81] \\
\hline \multirow{2}{*}{ Excoecaria agallocha } & Penicillium expansum 091006 & 海南文昌 & [82-83] \\
\hline & Penicillium sp. Y2-2-1 & 广东广州 & [84] \\
\hline \multirow{3}{*}{ Bruguiera sexangula } & Penicillium sp. 091402 & 海南 & [85] \\
\hline & Penicillium sp. $303^{\#}$ & 广东湛江 & {$[86]$} \\
\hline & Penicillium variabile & 福建 & [87] \\
\hline
\end{tabular}

$a /$ : No collection place.

\section{2 红树来源青霉属内生真菌次级代谢产物与生 物活性研究}

文献统计表明, 近 14年(2007 2020年)从红树来源 青霉属真菌中分离鉴定出的 276 个新的次级代谢产物主 要包括生物碱类、肽类、萜类及聚酮类等, 其中 140 个 化合物具有抗菌、抗肿瘤和杀虫等生物活性.

\section{1 生物碱类化合物}

已从红树来源青霉属内生真菌的代谢产物中分离 鉴定出生物碱类化合物 69 个(1 69) (2007 2020年)(图 1 和 2), 主要包括二酮哌嗪类生物碱、吲哚类生物碱及 其他类生物碱, 其中 6 个为新颖骨架结构的化合物 $(\mathbf{1 6}$, $17,20,21,64,66), 32$ 个生物碱类化合物具有抗菌、细 胞毒、抗氧化、杀虫及乙酰胆碱酯酶等生物活性(表 2).

从红树植物白骨壤(A. marina) 来源的真菌 P. brocae MA-231 培养液中分离鉴定出 5 个新的硫代二酮哌嗪生 物碱 penicibrocazines $\mathrm{A} \sim \mathrm{E}(\mathbf{1} \sim \mathbf{5})^{[44]}$ 和 6 个新的二硫键 桥联二酮哌嗪类生物碱 brocazines $A \sim F(\mathbf{6} \sim 11)^{[45]}$. 其 中硫代二酮哌嗪生物碱类化合物 $2 \sim 4$ 均对金黄色葡萄 球菌(Staphylococcus aureus) 有抑制活性, 其最低抑菌浓 度(MIC)分别为 $32.0,0.25,8.0$ 和 $0.25 \mu \mathrm{g} / \mathrm{mL}$; 化合物 3 对藤黄微球菌(Micrococcus luteus) 有较强的抑制活性, 其 MIC 值为 $0.25 \mu \mathrm{g} / \mathrm{mL}$; 化合物 $\mathbf{1} \sim \mathbf{3}$ 和 5 均对植物病 原菌小麦全蚀病菌(Gaeumannomyces graminis) 有抑制 活性, 其 MIC 值分别为 $0.25,8.0,0.25$ 和 $64.0 \mu \mathrm{g} / \mathrm{mL}$; 硫 代二酮哌嗪生物碱对多种人类致病菌和植物病原菌显 示较好的抑制活性, 初步构效关系研究表明, C-6 和 C-6'
为双键时能够增强对 $S$. aureus 的抑菌活性; $S$-甲基基团 及 C-5/5'位的羰基能够增强对 G. graminis 的抑制活性. 二硫键桥联二酮哌嗪生物碱类化合物 6, 7, 10 和 11 对 9 种肿瘤细胞 DU145, HeLa, HepG2, MCF-7, NCI-H460, SGC-7901, SW1990, SW480 和 U251 均具有较强的细胞 毒活性，其 $\mathrm{IC}_{50}$ 值为 $0.89 \sim 9.0 \mu \mathrm{mol} / \mathrm{L}$. 结果表明，当二 酮哌嗪类生物碱存在二硫键且为 $\alpha / \alpha^{\prime}$ 构型时具有显著的 细胞毒活性. 构效关系研究表明, C-6 和 C-6'双键为活性 必需基团, C-6 和 C-6 都为双键时的细胞毒活性强于只 有一个双键的活性. 此后, 继续从该菌中分离得到 4 个 新的硫代二酮哌嗪类生物碱 penicibrocazines $\mathrm{F} \sim \mathrm{I}$ $(12 \sim 15)^{[47]}$ 和 4 个新的二酮哌嗪类生物碱 spirobrocazines $\mathrm{A} \sim \mathrm{C}(\mathbf{1 6} \sim \mathbf{1 8})$ 和 brocazine $\mathrm{G}(\mathbf{1 9})^{[46]}$, 化合物 16 和 17 为骨架新颖的化合物, 均具有 6/5/6/5/6 的环系 统，且在 C-2 处有一个罕见的螺环中心; 其中化合物 $\mathbf{1 4}$ 对大肠杆菌(Escherichia coli)、嗜水气单胞菌(Aeromonas hydrophilia) 和副溶血弧菌( $V$. parahaemolyticus) 有中等 强度的抑制活性，其 MIC 值分别为 16.0, 32.0 和 16.0 $\mu \mathrm{g} / \mathrm{mL}$; 化合物 $\mathbf{1 4}$ 和 $\mathbf{1 5}$ 对哈维氏弧菌(Vibrio harveyi) 具 有一定的抑制活性，其 MIC 值分别为 16.0 和 32.0 $\mu \mathrm{g} / \mathrm{mL}$. 化合物 16 对大肠杆菌 (E. coli)、金黄色葡萄球菌 (S. aureus) 和哈维氏弧菌 (V.harveyi) 具有中等强度的抑 制活性，其 MIC 值分别为 32.0、16.0 和 $64 \mu \mathrm{g} / \mathrm{mL}$; 化合 物 18 对大肠杆菌 (E. coli)、嗜水气单胞菌 (A. hydrophilia) 和哈维氏弧菌 (V. harveyi) 具有抑制活性, 其 MIC 值均为 $32.0 \mu \mathrm{g} / \mathrm{mL}$; 化合物 19 对金黄色葡萄球菌 (S. aureus) 具 有较强的抑制活性, MIC 值为 $0.25 \mu \mathrm{g} / \mathrm{mL}$. 从红树植 

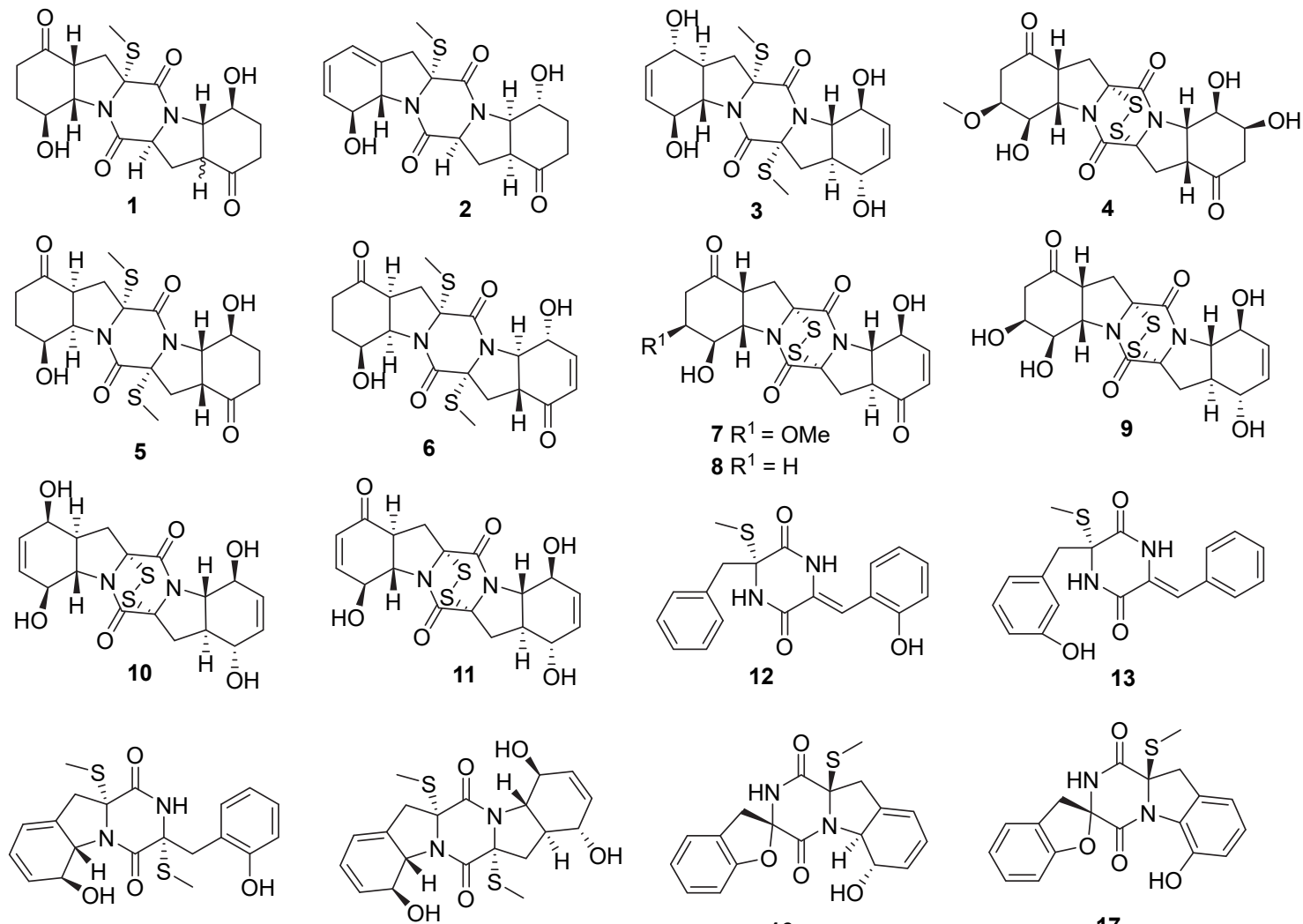

16

17

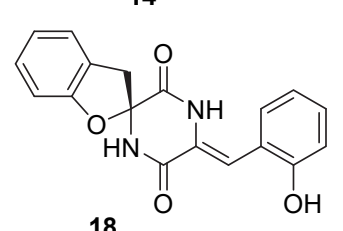

15

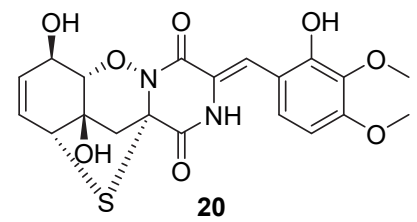

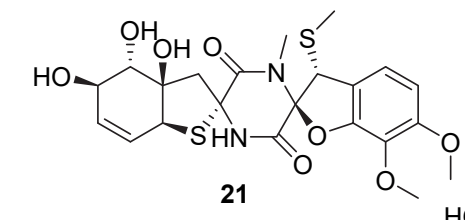
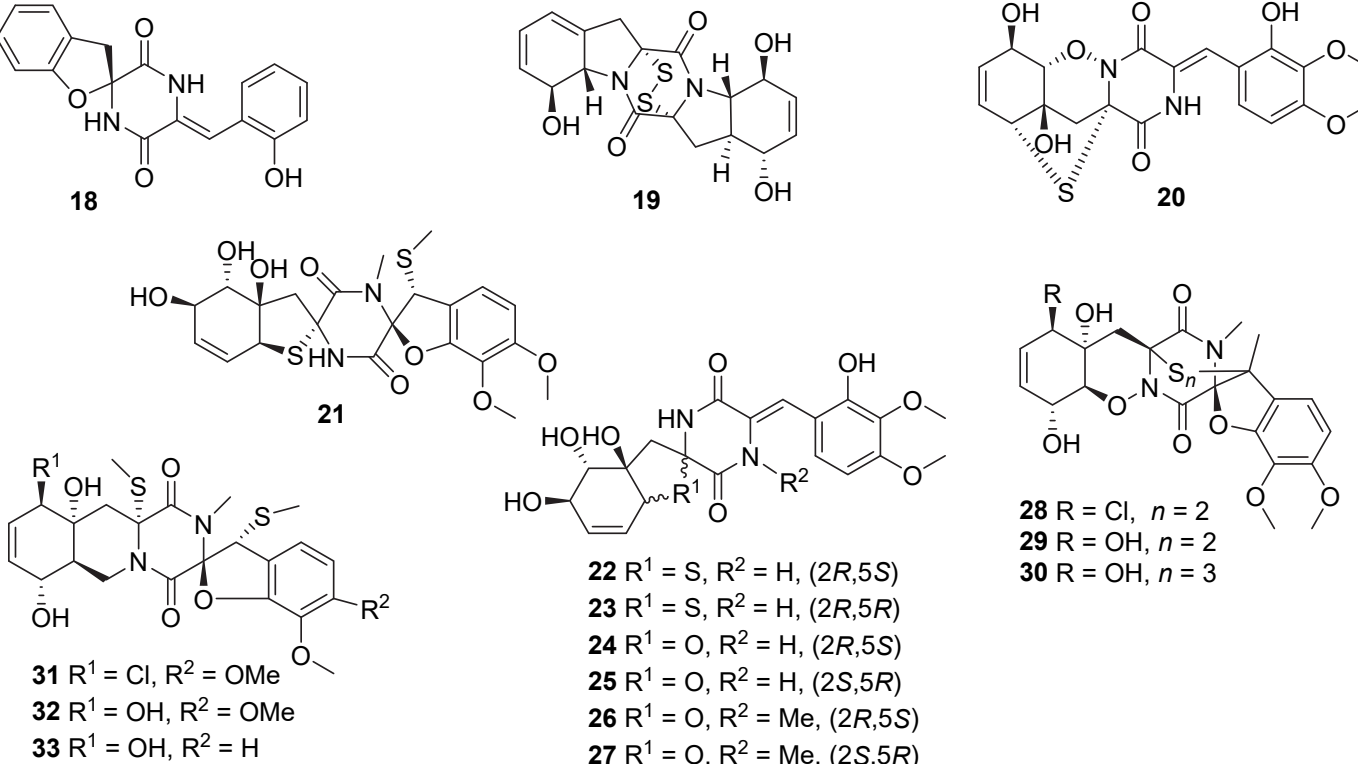

$22 \mathrm{R}^{1}=\mathrm{S}, \mathrm{R}^{2}=\mathrm{H},(2 R, 5 \mathrm{~S})$

$23 \mathrm{R}^{1}=\mathrm{S}, \mathrm{R}^{2}=\mathrm{H},(2 R, 5 R)$

$24 \mathrm{R}^{1}=\mathrm{O}, \mathrm{R}^{2}=\mathrm{H},(2 R, 5 \mathrm{~S})$

$25 \mathrm{R}^{1}=\mathrm{O}, \mathrm{R}^{2}=\mathrm{H},(2 \mathrm{~S}, 5 \mathrm{R})$

$26 \mathrm{R}^{1}=\mathrm{O}, \mathrm{R}^{2}=\mathrm{Me},(2 R, 5 S)$

$27 \mathrm{R}^{1}=\mathrm{O}, \mathrm{R}^{2}=\mathrm{Me},(2 S, 5 R)$

$29 \mathrm{R}=\mathrm{OH}, n=2$

$30 \mathrm{R}=\mathrm{OH}, n=3$

图 1 红树来源青霉属真菌产生的生物碱类化合物 $1 \sim 33$

Figure 1 Alkaloids $1 \sim 33$ produced by mangrove-derived Penicillium $\mathrm{sp}$.

物海桑(S. caseolaris)的 P. janthinellum HDN13-309 内生 真菌发酵提取物中分离出了 8 个新的二酮哌嗪类生物 碱 penispirozines $A \sim \mathrm{H}(\mathbf{2 0} \sim \mathbf{2 7})^{[67]}$, 其中化合物 20 具有 一个罕见的的吡嗪并 $[1,2]$ 噁杂䒺与噻吩环偶联的体系; 化合物 21 具有一个 6/5/6/5/6 五环系统和两个罕见的螺 环中心; 化合物 22 27 为具有手性的螺硫代或螺呋喃
环系统; 且化合物 22 和 23 在 $10 \mu \mathrm{mol} / \mathrm{L}$ 下增加了两种 相关的 II 期解毒酶(SOD2 和 HO-1)的表达. 从来源于红 树植物海桑 $(S$. caseolaris $)$ 的内生真菌 P. janthinellum HDN13-309 的发酵提取物中分离得到了 6 个新型的二

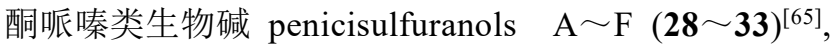
化合物 28 30 对人宫颈癌细胞 HeLa 和白血病原髓细 


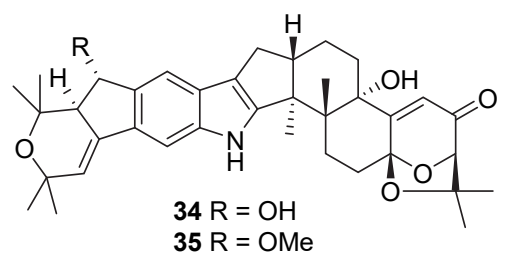

$>_{39}^{c}$

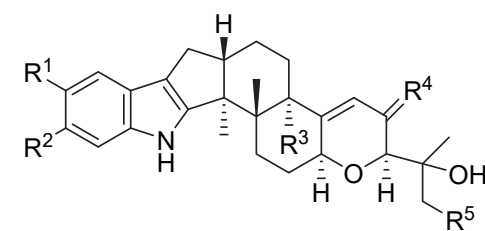

$42 \mathrm{R}^{1}=\mathrm{R}^{2}=\mathrm{R}^{3}=\mathrm{R}^{5}=\mathrm{H}, \mathrm{R}^{4}=\mathrm{H}_{2}$

$43 \mathrm{R}^{1}=\mathrm{R}^{2}=\mathrm{H}, \mathrm{R}^{3}=\mathrm{R}^{5}=\mathrm{OH}, \mathrm{R}^{4}=\mathrm{O}$

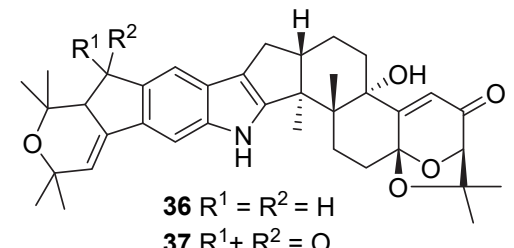

$37 R^{1}+R^{2}=0$
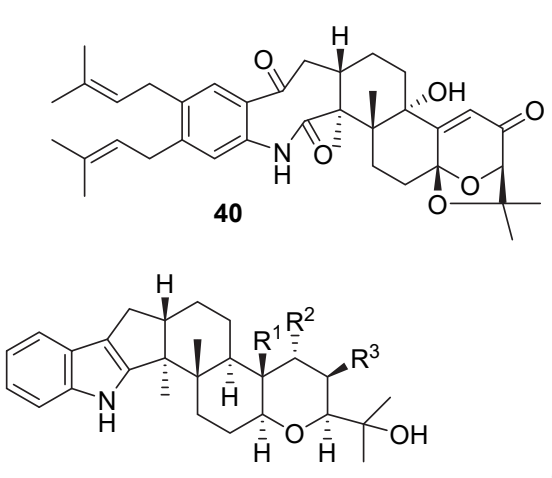

$45 \mathrm{R}^{1}=\mathrm{CO}_{2} \mathrm{H}, \mathrm{R}^{2}=\mathrm{R}^{3}=\mathrm{H}$

$46 R^{1}=R^{2}=R^{3}=H$

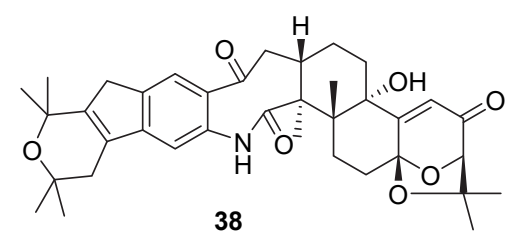

38

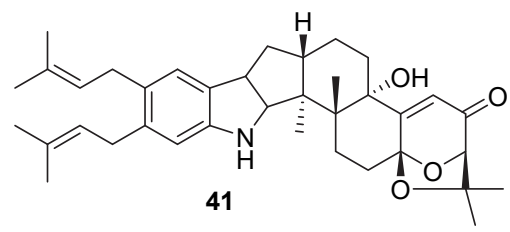<smiles>CC(C)=CCCC1(O)CCC(=CCc2c[nH]c3ccccc23)CC1</smiles>

47

$44 \mathrm{R}^{1}=\mathrm{R}^{2}=$ 公 $, \mathrm{R}^{3}=\mathrm{OH}, \mathrm{R}^{4}=\mathrm{O}, \mathrm{R}^{5}=\mathrm{H}$

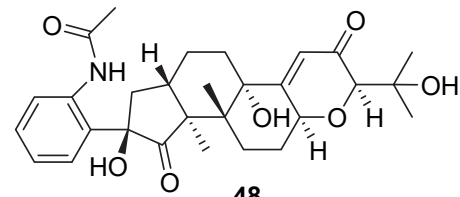

(c)

49<smiles>C[C@@H]1CN2C[C@H]3Cc4c([nH]c5ccccc45)C(C)(C)[C@H]3C[C@]23CC(=O)N[C@H]13</smiles>

53

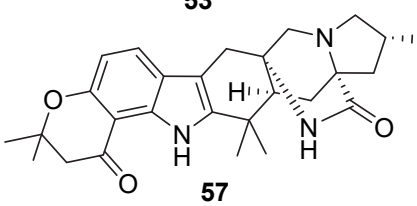

57<smiles>C=CC(C)(C)c1[nH]c2ccccc2c1CCNC(C)=O</smiles>

62

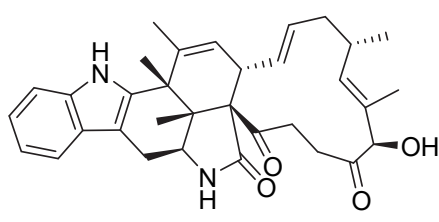

66

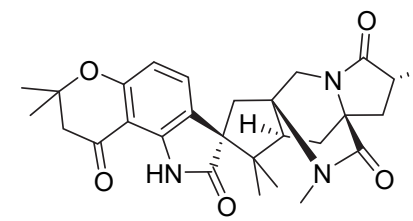
50

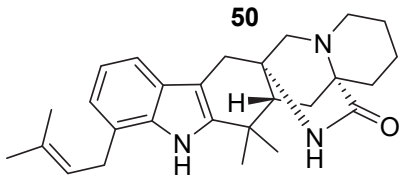

54<smiles>[R16]C1(O)C(=O)N2CCC[C@H]2[C@]1([R2])c1ccc(O)cc1</smiles>

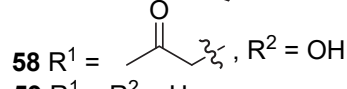

$59 \mathrm{R}^{1}=\mathrm{R}^{2}=\mathrm{H}$<smiles>C[C@@H]1C(=O)N2CCC[C@]2(CO)[C@@H]1O</smiles>

$\mathrm{OH}$

63

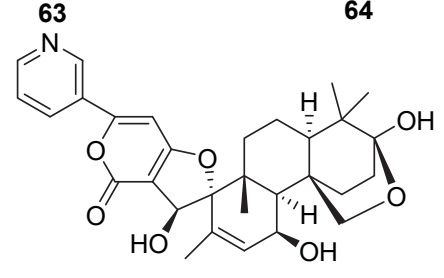

67

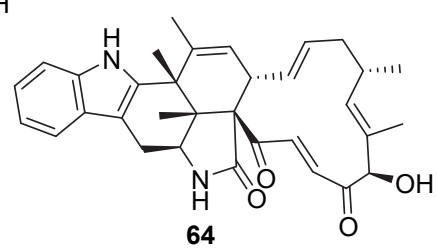

$\overbrace{1}^{(1)}$

68
(N)

$51 \quad 52$

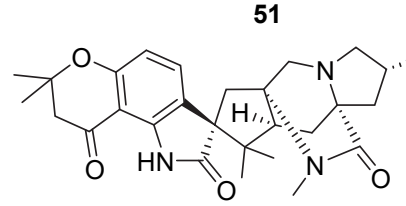

55

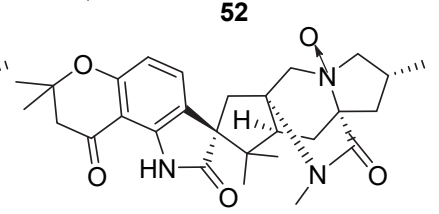

图 2 红树来源青霉属真菌产生的生物碱类化合物 34 69

Figure 2 Alkaloids 34 $\sim \mathbf{6 9}$ produced by mangrove-derived Penicillium sp. 
表 2 新生物碱类化合物 $\mathbf{1} \sim 69$ 的生物活性与产生菌的来源 ${ }^{a}$

Table 2 Biological activities of new alkaloids $1 \sim 69$ and the resources of producing strains

\begin{tabular}{|c|c|c|c|c|}
\hline Compound & Source & Bioactivity (MIC, $\left.\mathrm{LD}_{50}, \mathrm{IC}_{50}\right)$ & Region & Year \\
\hline $1 \sim 5$ & A. marina & $\begin{array}{l}\text { S. aureus: } 0.25 \sim 32.0 \mu \mathrm{g} / \mathrm{mL} \text { (except } 4 \text { ); } \\
\text { Gaeumannomyces graminis: } 0.25 \sim 64.0 \mu \mathrm{g} / \mathrm{mL} \text { (except } 4 \text { ) } \\
\text { Micrococcus luteus: } 0.25 \mu \mathrm{g} / \mathrm{mL}(\mathbf{3})\end{array}$ & Hainan & $2015^{[44]}$ \\
\hline $6 \sim 11$ & A. marina & $\begin{array}{l}\text { DU145/HeLa/HepG2/MCF-7/NCI-H460/SGC-7901/ } \\
\text { SW1990/SW480/U251: 0.89 9 } \mu \text { mol/L (except 8/9) }\end{array}$ & Hainan & $2014^{[45]}$ \\
\hline $12 \sim 15$ & A. marina & $\begin{array}{l}\text { E. coli/A. hydrophilia/V. harveyi/V. parahaemolyticus: } \\
16.0 \sim 32.0 \mu \mathrm{g} / \mathrm{mL}(\mathbf{1 4} / \mathbf{1 5})\end{array}$ & Hainan & $2017^{[47}$ \\
\hline $16 \sim 18$ & A. marina & $\begin{array}{l}\text { E. coli/A. hydrophilia/V. harveyi: } 16.0 \sim 64.0 \mu \mathrm{g} / \mathrm{mL}(\mathbf{1 6}) \text {; } \\
\text { E. coli/A. hydrophilia/V. harveyi: } 32 \mu \mathrm{g} / \mathrm{mL}(\mathbf{1 8})\end{array}$ & Hainan & $2016^{[46]}$ \\
\hline 19 & A. marina & S. aureus: $0.25 \mu \mathrm{g} / \mathrm{mL}$ & Hainan & $2016^{[44]}$ \\
\hline $\mathbf{2 0} \sim 27$ & Root of S. caseolaris & $\mathrm{SOD} 2 / \mathrm{HO}-1: 10 \mu \mathrm{mol} / \mathrm{L}(\mathbf{2 2} / \mathbf{2 3})$ & Hainan & $2020^{[67}$ \\
\hline $28 \sim 33$ & Root of $S$. caseolaris & $\begin{array}{l}\text { HeLa: } 0.3 \sim 3.9 \mu \mathrm{mol} / \mathrm{L}(\mathbf{2 8} \sim \mathbf{3 0}) \\
\text { HL-60: } 0.1 \sim 1.6 \mu \mathrm{mol} / \mathrm{L}(\mathbf{2 8} \sim \mathbf{3 0})\end{array}$ & Hainan & $2017^{[65}$ \\
\hline $34 \sim 41$ & Stem of $A$. corniculatum & $\mathrm{BK}_{\mathrm{Ca}}:(\mathbf{3 4}, \mathbf{3 5}, \mathbf{3 7})$ & Fujian & $2007^{[63]}$ \\
\hline 42 & Rhizospheric soil of mangrove plant & $\mathrm{H} 1 \mathrm{~N} 1: 28.3 \mu \mathrm{mol} / \mathrm{L}$ & Hainan & $2013^{[14]}$ \\
\hline 43 & Rhizospheric soil of mangrove Plant & $\mathrm{H} 1 \mathrm{~N} 1: 38.9 \mu \mathrm{mol} / \mathrm{L}$ & Hainan & $2013^{[14]}$ \\
\hline 44 & Rhizospheric soil of mangrove plant & $\mathrm{H} 1 \mathrm{~N} 1: 32.2 \mu \mathrm{mol} / \mathrm{L}$ & Hainan & $2013^{[14]}$ \\
\hline 45 & Rhizospheric soil of mangrove plant & / & Hainan & $2013^{[14]}$ \\
\hline 46 & Rhizospheric soil of mangrove plant & $\mathrm{H} 1 \mathrm{~N} 1: 73.3 \mu \mathrm{mol} / \mathrm{L}$ & Hainan & $2013^{[14]}$ \\
\hline 47 & Rhizospheric soil of mangrove plant & $\mathrm{H} 1 \mathrm{~N} 1: 34.1 \mu \mathrm{mol} / \mathrm{L}$ & Hainan & $2013^{[14]}$ \\
\hline 48 & Rhizospheric soil of mangrove plant & / & Hainan & $2018^{[16]}$ \\
\hline 49 & Rhizospheric soil of mangrove plan & l & Hainan & $2020^{[5]}$ \\
\hline $50 \sim 53$ & Rhizospheric soil of mangrove plant & / & Hainan & $2017^{[11]}$ \\
\hline 54 & Leaves of $R$. stylosa & A. salina: $5.6 \mu \mathrm{mol} / \mathrm{L}$ & Hainan & $2015^{[58]}$ \\
\hline $55 \sim 57$ & Rhizospheric soil of mangrove plant & AchE: $58.0 \mu \mathrm{mol} / \mathrm{L}(\mathbf{5 7})$ & Hainan & $2014^{[13]}$ \\
\hline 58,59 & A. marina & S. aureus: $0.125 \mu \mathrm{g} / \mathrm{mL}$ (58) & Hainan & $2017^{[43]}$ \\
\hline $60 \sim 62$ & Leaves of $A$. marina & A. salina: $36.7 \mu \mathrm{mol} / \mathrm{L}$ & Hainan & $2014^{[49]}$ \\
\hline 63 & B. gymnorrhiza & l & Hainan & $2014^{[38]}$ \\
\hline $64 \sim 66$ & M. bontioides & $\begin{array}{l}\text { Rhizoctonia solani: } 195.98 \mu \mathrm{mol} / \mathrm{L}(\mathbf{6 4}, \mathbf{6 6}) \\
\text { MTA-MB435/A549: } 7.32 \sim 16.13 \mu \mathrm{mol} / \mathrm{L}\end{array}$ & Leizhou Peninsula & $\begin{array}{l}2017^{[74]} \\
2016^{[75]}\end{array}$ \\
\hline 67 & A. ilicifolius & / & Hainan & $2015^{[56]}$ \\
\hline 68 & Leaves of $R$. stylosa & A. salina: $2.3 \mu \mathrm{mol} / \mathrm{L}$ & Hainan & $2015^{[58]}$ \\
\hline 69 & Branch of C. manghas & 1 & Hainan & $2015^{[60]}$ \\
\hline
\end{tabular}

胞 HL-60 显示出强的抑制活性, 对 HeLa 的 $\mathrm{IC}_{50}$ 值分别 为 $0.3,0.5,3.9 \mu \mathrm{mol} / \mathrm{L}$, 对 $\mathrm{HL}-60$ 的 $\mathrm{IC}_{50}$ 值分别为 0.1 , $1.2,1.6 \mu \mathrm{mol} / \mathrm{L}$, 构效关系研究表明二硫桥键为重要的 活性基团. 查阅文献发现硫代二酮哌嗪生物碱主要从药 用植物仪花(lysidice rhodostegia)、冬虫夏草、二道柳 (Salix sp.) 和深海来源的附球霉属真菌 Epicoccum nigrum、海洋来源的茎点霉属真菌 Phoma sp. 中分离得 到[88-92], 从红树来源青霉属真菌中分离获得了 4 个 $(\mathbf{1 6}$, 17, 20 和 21) 骨架结构新渘硫代二酮哌嗪类化合物, 他 们螺环结构或者具有吡嗪并 $[1,2]$ 噁杂菜与噻吩环偶联 的骨架结构.

吲哚生物碱广泛存在于海洋软体动物、三七等药 用植物及药用红树来源的青霉属真菌 Penicillium sp.、
掘氏梅里属 Drechmeria sp. 和毛霉属 Mucor sp. 真菌等, 具有酶抑制剂、细胞毒及抗菌等生物活性 ${ }^{[93-98]}$. 其中, 从红树植物桐花树 (A. corniculatum) 的内生真菌 Penicillium sp. (strain HKI0459)中分出了 8 个新的吲哚 生物碱 shearinines $\mathrm{D} \sim \mathrm{K}(\mathbf{3 4} \sim \mathbf{4 1})^{[63]}$, 化合物 34, 35 和 37 显示强的 $\mathrm{BK}_{\mathrm{Ca}}$ 通道抑制作用, 其 $\mathrm{IC}_{50}$ 值为 150 和 170 $\mathrm{nmol} / \mathrm{L}$. 从来源于红树林根际土壤的内生真菌 $P$. camemberti OUCMDZ-1492 中分离出了 7 个新的吲哚二

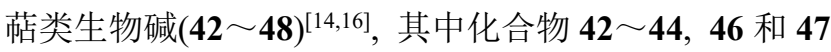
均对 H1N1 病毒表现出显著的抑制活性，其 $\mathrm{IC}_{50}$ 值分 别为 $28.3,38.9,32.2,73.3,34.1 \mu \mathrm{mol} / \mathrm{L}$. 从来源于红树 林根际土壤来源真菌 P. janthinellum HK1-6 中分离获 得了 1 个新的吲哚生物 paraherquamide $J(49)^{[5]}$. 从红 
树沉积物来源真菌 Penicillium sp. SCSIO041218 中分 离获得了 4 个新的吲哚生物碱 $(\mathbf{5 0} \sim \mathbf{5 3})^{[11]}$. 从红树植 物红海榄 (R. stylosa) 的内生真菌 P. oxalicum EN-201 的 大米发酵提取物中分离出了 1 个新的吲哚生物碱 penioxamide A (54) ${ }^{[58]}$, 该化合物显示出一定的卤虫 (A. salina) 致死活性, 其 $\mathrm{LD}_{50}$ 值为 $5.6 \mu \mathrm{mol} / \mathrm{L}$. 从红树沉 积物来源真菌 Penicillium sp. SYFz-1 的提取物中分离出 3 个新的吲哚生物碱 mangrovamides $\mathrm{A} \sim \mathrm{C}(\mathbf{5 5} \sim \mathbf{5 7})^{[9]}$, 其中化合物 57 具有中等强度的乙酰胆碱酯酶(AchE)抑 制性, $\mathrm{IC}_{50}$ 值为 $58.0 \mu \mathrm{mol} / \mathrm{L}$. 从来源于红树植物白骨壤 (A. marina) 的内生真菌 P. broca MA-231 的发酵提取物 中分离得到了 2 个新对羟基苯并吡咯嗪衍生物 brocapyrozins A 和 B $(\mathbf{5 8}, \mathbf{5 9})^{[47]}$, 其中化合物 $\mathbf{5 9}$ 对金黄 色葡萄球菌 (S. aureus) 有显著的抑制作用, MIC 值为 $0.125 \mu \mathrm{g} / \mathrm{mL}$.

此外, 从红树来源青霉属真菌中也分离获得了其他 类型的生物碱. 从来源于红树植物白骨壤(A. Marina) 的 内生真菌 P. brocae MA-192 中分离得到了 3 个不同于其 他生境来源的 C-2 位异戊烯基化的生物碱 brocaeloids $\mathrm{A} \sim \mathrm{C}(\mathbf{6 0} \sim \mathbf{6 2})^{[49]}$, 其中化合物 $\mathbf{6 1}$ 对卤虫 (A. salina) $)^{\text {表 }}$ 现出较强的致死活性, $\mathrm{LD}_{50}$ 值为 $36.7 \mu \mathrm{mol} / \mathrm{L}$. 从红树植 物木榄(B. gymnorrhiza)中分离的内生真菌 Penicillium sp. GD6 发酵提取物中分离出了 1 个新的吡咯生物碱 penibruguieramine A $(63)^{[38]}$. 从来源于半红树植物苦槛 蓝(M. bontioides)的内生真菌 P. chrysogenum V11 的固 体培养物中分离到了 3 个新的细胞松驰素 penochalasins $I \sim K(64 \sim 66)^{[74-75]}$, 不同于其他生境来源的青霉属 真菌, 化合物 64、66 都是具有罕见的六环 6/5/6/5/6/13
稠环系统, 且对丝核菌(Rhizoctonia solani)均具有较弱 的抑制活性，其 MIC 值均为 $195.98 \mu \mathrm{mol} / \mathrm{L}$; 化合物 64 和 66 均对 MTA-MB 435, SGC-7901 和 A549 肿瘤细胞 株均具有明显的生长抑制活性，其 $\mathrm{IC}_{50}$ 为 $7.32 \sim 16.13$ $\mu \mathrm{mol} / \mathrm{L}$; 化合物 $\mathbf{6 5}$ 对炭疽菌(Colletotrichum gloeosporioides) 显示强的抑制活性, 其 MIC 值为 $25.08 \mu \mathrm{mol} / \mathrm{L}$. 从 来源于红树植物老鼠簕 (A. ilicifolius) 的内生真菌 Penicillium sp. $299^{\#}$ 的提取物中分离出了 1 个新的含 末端氰基的苯二氮类生物碱(67) ${ }^{[56]}$. 从来源于红树植 物红海榄 (R. stylosa) 的内生真菌 P. oxalicum EN-201 的 大米发酵提取物中分离出了 1 个新的癸醇类似物 decaturin analogue B (68) ${ }^{[58]}$, 化合物 $\mathbf{6 8}$ 显示出一定的 卤虫 (A. salina) 致死活性, 其 $\mathrm{LD}_{50}$ 值为 $2.3 \mu \mathrm{mol} / \mathrm{L}$. 从 来源于红树植物海芒果 (C. manghas) 的内生真菌 Penicillium sp. HN29-3B1 培养物中分离得到了 1 个新 化合物 penicidone D $(\mathbf{6 9})^{[60]}$.

\section{2 肽类化合物}

已从红树来源青霉属内生真菌的次级代谢产物中 分离鉴定出了肽类化合物 11 个 $(\mathbf{7 0} \sim \mathbf{8 0})(2015 \sim 2020$ 年) (图 3), 其中 1 个化合物(72)为骨架新颖的肽类化合 物, 4 个肽类化合物具有抗菌、抗氧化等生物活性(表 3).

从红树植物苦槛蓝(Myoporum bontioides)的内生真 菌 P. chrysogenum $\mathrm{V} 11$ 中分离的到了 1 个新的 $L$-丙氨酸 衍生物 $N$-fumaryl- $L$-alanine dimethyl ester (70)和 1 个新 天然产物 $N, N^{\prime}$-bis[(S)-1-methoxycarbonylethyl] fumaric diamide (71) ${ }^{[73]}$. 从红树植物木榄 (B. gymnorrhiza) 的内 生真菌 Penicillium sp. GD6 培养物中分离得到了 1 个在

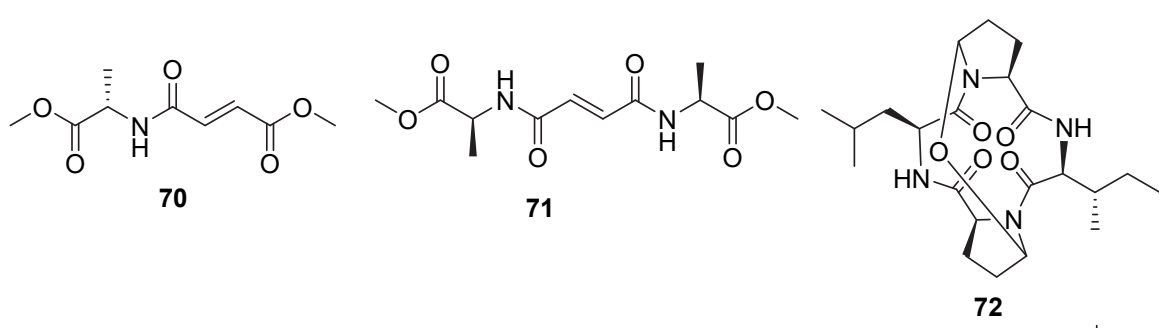
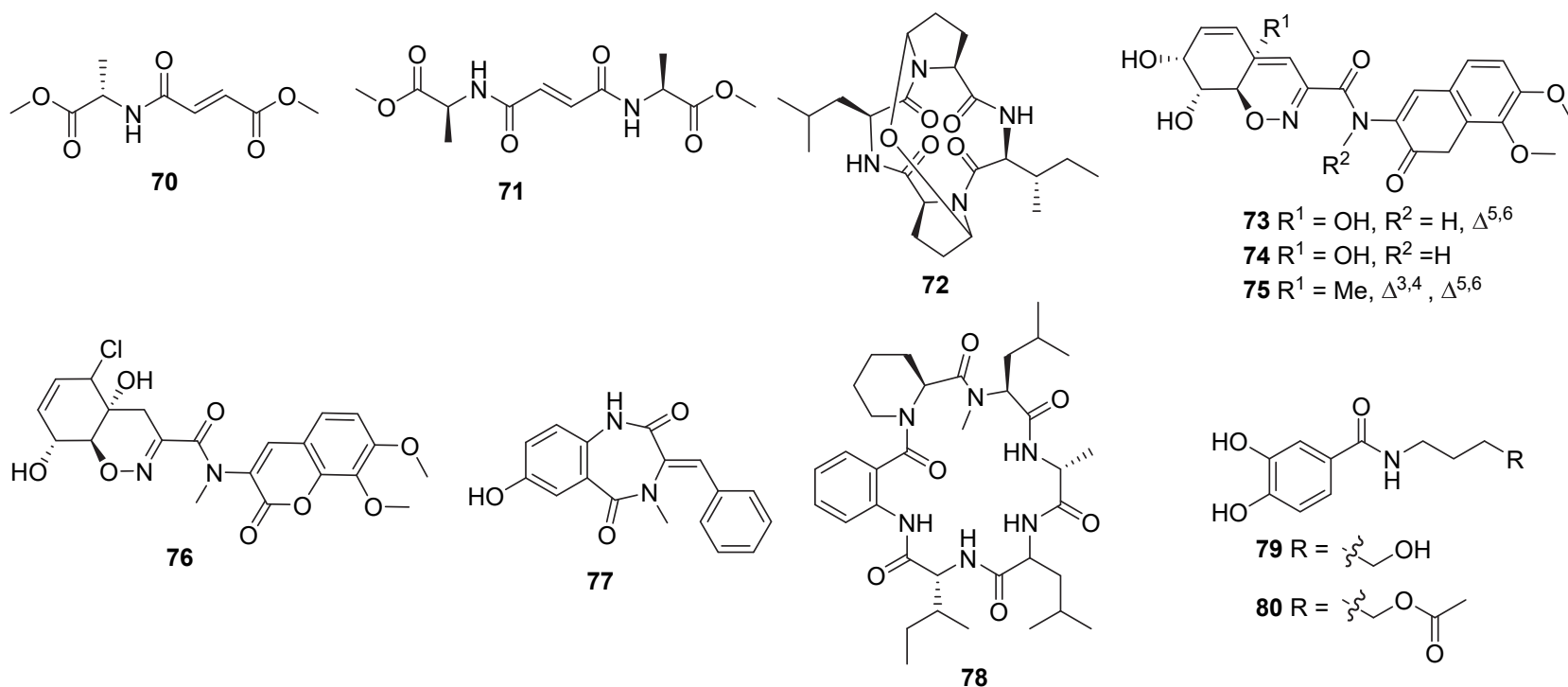

图 3 红树来源青霉属真菌产生的肽类化合物 $70 \sim 80$

Figure 3 Peptides $\mathbf{7 0} \sim \mathbf{8 0}$ produced by mangrove-derived Penicillum sp. 
表 3 新肽类化合物 $\mathbf{7 0} \sim \mathbf{8 0}$ 的生物活性与产生菌的来源 ${ }^{a}$

Table 3 Biological activities of new peptides $\mathbf{7 0} \sim \mathbf{8 0}$ and the resources of producing strains

\begin{tabular}{|c|c|c|c|c|}
\hline Compound & Source & Bioactivity (MIC, LD $\mathrm{LD}_{50}, \mathrm{IC}_{50}$ ) & Region & Year \\
\hline 70 & M. bontioides & l & Leizhou Peninsula & $2018^{[73]}$ \\
\hline 71 & M. bontioides & / & Leizhou Peninsula & $2018^{[73]}$ \\
\hline 72 & Stem of $B$. gymnorrhiza & l & Guangdong & $2018^{[37]}$ \\
\hline $73 \sim 75$ & Root of S. caseolaris & / & Hainan & $2017^{[64]}$ \\
\hline 76 & Root of $S$. caseolaris & $\mathrm{H}_{2} \mathrm{O}_{2}$-induced oxidative damage & Hainan & $2017^{[66]}$ \\
\hline 77 & Rhizospheric soil of mangrove plant & 1 & Fujian & $2020^{[19]}$ \\
\hline 78 & Leaves of E. agallocha & $\begin{array}{l}\text { Salmonell paratyphi/E. coli/Shigella/ } \\
\text { V. parahaemolyticus: } 50 \mathrm{mg} / \mathrm{mL}\end{array}$ & Guangdong & $2020^{[84]}$ \\
\hline 79,80 & Soil of $R$. stylosa & DPPH: 5.6 (79), $4.7 \mu \mathrm{mol} / \mathrm{L}$ (80) & 1 & $2015^{[59]}$ \\
\hline
\end{tabular}

a/: No activity.

$\mathrm{C}(5)-\mathrm{C}\left(5^{\prime}\right)$ 之间含有醚桥的环四肽 5,5'-epoxyMKN-349A (72) ${ }^{[37]}$. 从红树植物海桑 (S. caseolaris) 的 内生真菌 P. janthinellum HDN13-309 的发酵液中分离 得到了 3 个具有高度氧化的 1,2-吘唑烷核的杂环二肽

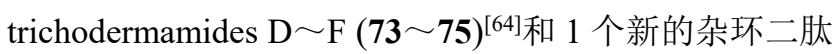
$N$-methyl-trichodermamide B (76) ${ }^{[66]}$, 其中化合物 76 对 $\mathrm{H}_{2} \mathrm{O}_{2}$ 诱导的氧化损伤具有细胞保护作用. 从红树林沉 积物来源的真菌 P. polonicum MCCC3A00951 的培养液 中分离了 1 个新的化合物 7-hydroxy-3,10-dehydrocyclopeptine (77) ${ }^{[19]}$. 从红树植物海漆 (E. agallocha) 来源的内 生真菌 Penicillium sp. Y2-2-1 的粗提物分离出了 1 个新 的六肽类化合物 Anthranicine (78) $)^{[84]}$, 且该化合物在 50 $\mathrm{mg} / \mathrm{mL}$ 浓度下对四种食源性致病菌副伤寒沙门氏菌 (Salmonell paratyphi)、大肠杆菌 (E. coli)、志贺氏菌 (Shigella)、副溶血弧菌 $(V$. parahaemolyticus $)$ 均具有显 著的抑制活性. 从红树植物红海榄 (R. stylosa) 的内生真 菌 P. solitum GWQ-143 中分离得到了 2 个新的肽类化

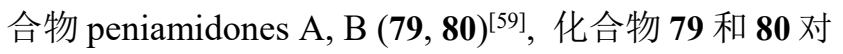
1,1-二苯基-2-三硝基苯肼(DPPH)具有较强的清除自由 基活性，其 $\mathrm{IC}_{50}$ 值分别为 5.6 和 $4.7 \mu \mathrm{mol} / \mathrm{L}$.

\section{3 萜类化合物}

已从红树来源青霉属内生真菌的代谢产物中分离 鉴定出了萜类化合物 26 个 $(81 \sim 106)(2010 \sim 2020$ 年)(图 4), 其中 4 个化合物为具有新颖骨架的化合物 $(\mathbf{8 9}, \mathbf{9 0}$, $93,105), 12$ 个萜类化合物具有抗菌、细胞毒、杀虫、 $\alpha$ 葡萄糖苷酶、乙酰胆碱酷酶等生物活性(表 4).

从红树植物尖瓣海莲 (B. Sexangula var. rhyn(chopetala) 的内生真菌 P. simplicissimum MA-332 中分离 出了 1 个结构新颖的混源萜类化合物 simpterpenoid A $(\mathbf{8 1})^{[35]}$, 化合物 81 对梨状梨轮纹病菌 (Physalospora piricola) 具有中度的抑制活性, 其 MIC 值为 $64 \mu \mathrm{g} / \mathrm{mL}$. 从红树植物角果木 (C. tagal) 的内生真菌 Penicillium sp. $\mathrm{J}-54$ 的发酵液中分离得到了 7 个新没药烷型倍半萜 penicieudesmols $A \sim G(\mathbf{8 2} \sim \mathbf{8 8})^{[2,68]}$, 化合物 83 对肿瘤细 胞 K-562 具有较弱的细胞毒活性, 其 $\mathrm{IC}_{50}$ 值为 90.1 $\mu \mathrm{mol} / \mathrm{L}$; 化合物 87 具有用 $\alpha$-葡萄糖苷酶抑制活性, 其 $\mathrm{IC}_{50}$ 值为 $2.27 \pm 0.058 \mathrm{mmol} / \mathrm{L}$. 从红树植物榄李 $(L$. racemosa) 的内生真菌 P. bilaiae MA-267 中分离鉴定出 了 2 个结构新㖪的具有三环 $\left[6.3 .1 .0^{1,5}\right]$ 十二烷骨架的倍 半萜类化合物 penicibilaenes A, B $(\mathbf{8 9}, \mathbf{9 0})^{[71]}$, 化合物 89 和 90 均对植物病原菌炭疽病菌 (C. gleosporioides) 有明 显的抑制作用, 其 MIC 分别为 1.0 和 $0.125 \mu \mathrm{g} / \mathrm{mL}$. 从红 树植物海漆 (E. agallocha) 的内生真菌 P. expansum 091006 的提取物中分离出了 2 个新的没药烷型倍半萜 类化合物 $(S)-(+)$-11-dehydrosydonic acid (91) 和 $(7 S, 11 S)$ $(+)$-12-acetoxysydonic acid $(\mathbf{9 2})^{[82]}$.

从红树林植物尖瓣海莲 (B. Sexangula var. rhynchopetala $)$ 的内生真菌 Penicillium sp. TGM112 中分离 出了 2 个新的混源萜类化合物 penicianstinoids A, B (93,

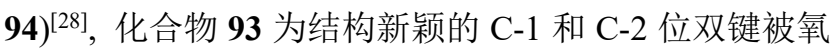
化为羰基的混源萜类化合物，化合物 93 和 94 对线虫 $(C$. elegans) 具有杀虫活性, 其 $\mathrm{EC}_{50}$ 值分别为 9.4 和 9.9 $\mu \mathrm{g} / \mathrm{mL}$. 从红树植物海莲 (B. sexangula) 的内生真菌 Penicillium sp. $303^{\#}$ 的代谢产物中分离出了 2 个新的混 源萜类化合物 $(95,96)^{[86]}$, 化合物 96 对人肿瘤细胞 MDA-MB 435, HepG2, HCT-116 和 A549 均具有一定的 生长抑制活性, 其 $\mathrm{IC}_{50}$ 值分别为 (31.32 2 2.63), (23.87 \pm 1.79$),(29.19 \pm 2.37)$ 和( $34.06 \pm 1.17) \mu \mathrm{mol} / \mathrm{L}$. 此 类混源萜类主要从曲霉属真菌 Aspergillus variecolor, $A$. ustus, A. nidulans, 青霉属真菌 P. diversum, P. purpurogenum、裸狍壳属 Emericella $\mathrm{sp}$. 和拟盘多毛狍属 Pestalotiopsis sp.等属的真菌中分离得到, 具有抗炎、杀 虫及抗菌等生物活性 ${ }^{[99-102]}$, 从红树来源青霉属真菌中 分离获得的部分混源萜具有更高的氧化度, 例如化合物 93 为结构新颖的 C-1 和 C-2 位双键被氧化为羰基的混源 萜类化合物。 


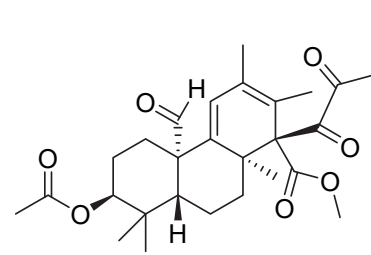

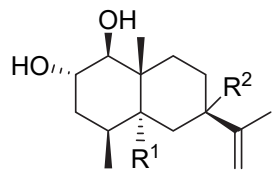

$82 \mathrm{R}^{1}=\mathrm{H}, \mathrm{R}^{2}=\mathrm{H}$ $83 \mathrm{R}^{1}=\mathrm{H}, \mathrm{R}^{2}=\mathrm{OH}$ $84 \mathrm{R}^{1}=\mathrm{OH}, \mathrm{R}^{2}=\mathrm{H}$ $85 \mathrm{R}^{1}=\mathrm{OH}, \mathrm{R}^{2}=\mathrm{OH}$

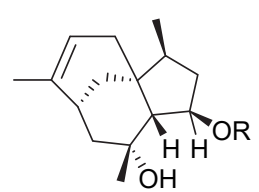

$\mathrm{HO}_{2} \mathrm{C}$<smiles>[R]CCCC(C)(O)c1ccc(C)cc1O</smiles>

$89 \mathrm{R}=\mathrm{H}$ $90 \mathrm{R}=\mathrm{Ac}$

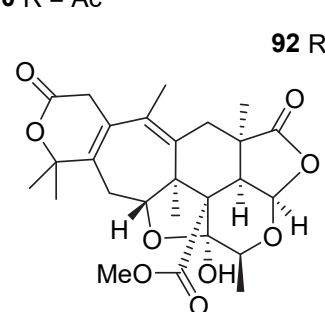

96<smiles>[13BH][13C](=C)C(=C)C</smiles>

$92 \mathrm{R}=$ ?

97<smiles>CC1C[C@H](O)C(O)[C@]2(C)CCC(C(C)(O)CO)C[C@@H]12</smiles>

86<smiles>CC1C[C@@H](O)C(O)[C@]2(C)CCC(C(C)(O)CO)C[C@]12O</smiles>

87<smiles>CC1C[C@@H](O)[C@@H](O)[C@@H]2CC[C@@H](C(C)CO)C[C@@H]12</smiles>

88

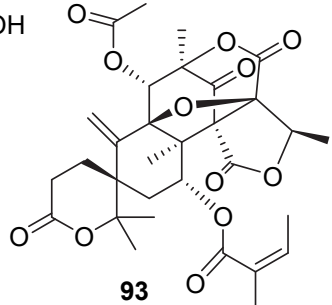

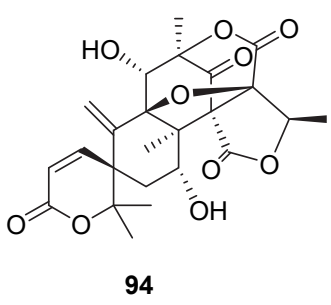

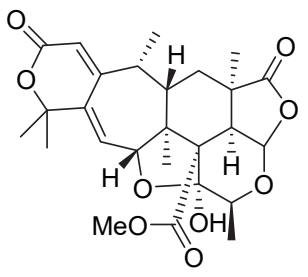

95

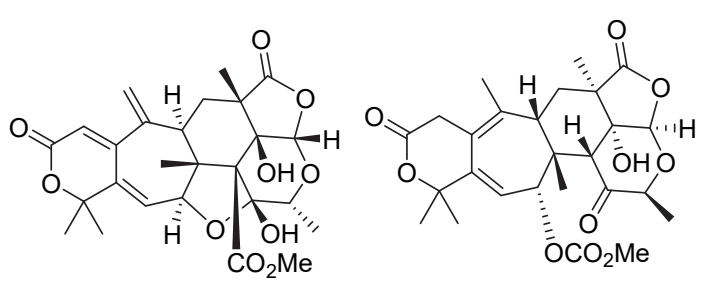

98

99<smiles>COc1ccc(-c2cc3c(c(=O)o2)CC2[C@](C)(CC[C@H]4C(C)(O)C(O)CC[C@]24C)O3)cc1</smiles><smiles>C=C(CC[C@H](C)[C@H]1CC=C2C3=C(C(=O)[C@H](OC(C)=O)[C@@]21C)[C@@]1(C)C[C@H](O)C(O)C(C)(C)C1CC3)C(C)C</smiles>

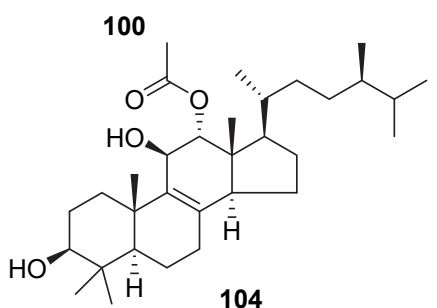

101<smiles>C=C(C)[C@H]1[C@@H](OC(C)=O)C[C@@H](C)[C@]12CC=C(C)[C@H](OC(=O)CCCCCCC/C=C\C/C=C\CCCCC)C2</smiles>

105

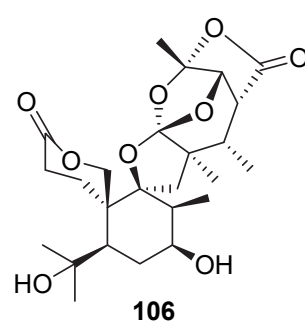

图 4 红树来源青霉属真菌产生的萜类化合物 $81 \sim 106$

Figure 4 Terpenoids $\mathbf{8 1} \sim \mathbf{1 0 6}$ produced by mangrove-derived Penicillum sp.

表 4 新萜类化合物 81 106 的生物活性与产生菌的来源 $a$

Table 4 Biological activities of new terpenoids $81 \sim 106$ and the resources of producing strains

\begin{tabular}{|c|c|c|c|c|}
\hline Compound & Source & Bioactivity (MIC, LD50, IC50) & Region & Year \\
\hline 81 & Soil of $B$. sexangula var. rhynchopetala & Physalospora piricola: $64 \mu \mathrm{g} / \mathrm{mL}$ & Hainan & $2018^{[35]}$ \\
\hline $82 \sim 88$ & Leaves of C. tagal & $\begin{array}{l}\text { K-562: } 90.1 \mu \mathrm{mol} / \mathrm{L}(\mathbf{8 3}) \\
\alpha \text {-glucosidase: } 2.27 \pm 0.058 \mu \mathrm{mol} / \mathrm{L}(\mathbf{8 7})\end{array}$ & Hainan & $2019^{[2,68]}$ \\
\hline 89,90 & L. racemose & C. gleosporioides: $0.125 \sim 1.0 \mu \mathrm{g} / \mathrm{mL}$ & Hainan & $2014^{[71]}$ \\
\hline 91 & Root of E. agallocha & 1 & Hainan & $2010^{[82]}$ \\
\hline 92 & Root of E. agallocha & l & Hainan & $2010^{[82]}$ \\
\hline 93,94 & B. sexangula var. rhynchopetala & C. elegans: $9.4 \sim 9.9 \mu \mathrm{g} / \mathrm{mL}$ & Hainan & $2019^{[28]}$ \\
\hline 95 & B. sexangular & $\begin{array}{l}\text { MDA-MB 435/HepG2/HCT-116/A549: } \\
23.87 \sim 34.06 \mu \mathrm{mol} / \mathrm{L}\end{array}$ & Guangdong & $2014^{[86]}$ \\
\hline 96 & B. sexangular & $\begin{array}{l}\text { MDA-MB } 435 / \mathrm{HepG} 2 / \mathrm{HCT}-116 / \mathrm{A} 549: \\
23.87 \sim 34.06 \mu \mathrm{mol} / \mathrm{L}\end{array}$ & Guangdong & $2014^{[86]}$ \\
\hline
\end{tabular}




\begin{tabular}{|c|c|c|c|c|}
\hline Compound & Source & Bioactivity $\left(\mathrm{MIC}, \mathrm{LD}_{50}, \mathrm{IC}_{50}\right)$ & Region & Year \\
\hline 97 & Soil of B. gymnorrhiza & 1 & Hainan & $2012^{[41]}$ \\
\hline 98 & Soil of $B$. gymnorrhiza & / & Hainan & $2012^{[41]}$ \\
\hline 99 & Soil of B. gymnorrhiza & / & Hainan & $2012^{[41]}$ \\
\hline 100 & Leaves of $K$. candel & l & Guangxi & $2016^{[23]}$ \\
\hline 101 & Laves of $K$. candel & AchE: $0.64 \pm 0.08 \mu \mathrm{mol} / \mathrm{L}$ & Guangxi & $2013^{[24]}$ \\
\hline 102 & Root of H. littoralis & 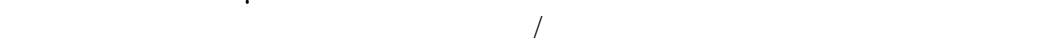 & Thailand & $2015^{[80]}$ \\
\hline 103 & $\begin{array}{l}\text { B. sexangula var. } \\
\text { rhynchopetala }\end{array}$ & $\begin{array}{l}\text { E. coli ATCC 25922/S. albus ATCC 8799/S. aureus ATCC 27154/ } \\
\text { B. cereus ATCC 11778: } 9.76 \mu \mathrm{mol} / \mathrm{L}(\mathbf{1 0 3})\end{array}$ & Hainan & $2014^{[36]}$ \\
\hline 104 & $\begin{array}{l}\text { B. sexangula var. } \\
\text { rhynchopetala }\end{array}$ & $\begin{array}{l}\text { E. coli ATCC } 25922 / S \text {. albus ATCC } 8799 / S \text {. aureus ATCC } 27154 / B \text {. ATCC } 663 / \\
\text { B. cereus ATCC } 11778 / \text { M. tetragenus ATCC } 13623): 5 \sim 10 \mu \mathrm{mol} / \mathrm{L} \mathrm{(104)}\end{array}$ & Hainan & $2014^{[36]}$ \\
\hline 105,106 & Soil of $L$. racemose & C. cornigerum, Edwardsiella tarda: $0.25,0.5 \mu \mathrm{mol} / \mathrm{L}$ (105) & Hainan & $2020^{[72]}$ \\
\hline
\end{tabular}

$a /$ : No activity.

从红树植物木榄 (B. gymnorrhiza) 的内生真菌 Penicillium sp. MA-37 中分离得到了 3 个单萜类衍生物 $(97 \sim 99)^{[41]}$. 从红树植物秋茄 $(K$. candel $)$ 的内生真菌 Penicillium sp. SK5GW1L 的代谢产物中分离得到了 2 个 $\alpha$-吡喃酮混源萜类化合物 3-epiarigsugacin E (100 $)^{[23]}$ 和 arigsugacin I (101) ${ }^{[24]}$, 化合物 101 对乙酰胆碱酯酶 $(A c h E)$ 有显著的抑制活性, $\mathrm{IC}_{50}$ 值为 $0.64 \pm 0.08 \mu \mathrm{mol} / \mathrm{L}$. 从红树植物银叶树 (H. littoralis) 的内生真菌 $P$. chermesinum strain HLit-OR2 的代谢产物分离出了 1 个 新化合物 $(\mathbf{1 0 2})^{[80]}$. 从红树植物尖瓣海莲 (B. Sexangula var. rhynchopetala) 的内生真菌 Penicillium sp. (J41221) 的乙酸乙酯粗提物中分离得到了 2 个三萜类化合物 $(103,104)^{[36]}$, 化合物 103 对 4 株致病菌大肠杆菌 (E. coli ATCC 25922)、白色葡萄球菌(S. albus ATCC 8799)、金 黄色葡萄球菌(S. aureus ATCC 27154) 和蜡状芽狍杆菌 (B. cereus ATCC 11778) 具有一定的抗菌活性, 其 MIC 值 均为 $9.76 \mu \mathrm{mol} / \mathrm{L}$. 化合物 $\mathbf{1 0 4}$ 对 6 株致病菌大肠杆菌 $(E$. coli ATCC 25922)、白色葡萄球菌(S. albus ATCC 8799)、 金黄色葡萄球菌(S. aureus ATCC 27154)、枯草芽孢杆菌 (B.ATCC 6633)、蜡状芽孢杆菌(B. cereus ATCC 11778) 及四联球菌(M. tetragenus ATCC 13623)均显示出一定的 抗菌活性, 其 MIC 值为 $5 \sim 10 \mu \mathrm{mol} / \mathrm{L}$. 从红树植物榄李 (L. racemosa) 的内生真菌 P. bilaiae MA-267 中分离鉴定 出了 2 个新的混源萜类化合物 chermebilaenes A, B (105,

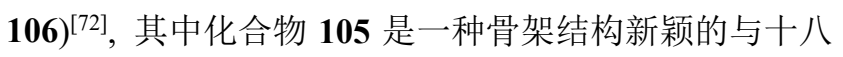
碳二烯酸骨架杂交的橡果烷型倍半萜类化合物, 对角质 角化藻菌(Ceratobasidium cornigerum) 和迟缓爱德华氏 菌(Edwardsiella tarda) 显示出很好的抗菌活性, 其 MIC 值分别为 0.25 和 $0.5 \mu \mathrm{mol} / \mathrm{L}$.

\section{4 聚酮类}

已从红树来源青霉属真菌的次级代谢产物中分离 鉴定出聚酮类化合物 140 个(107～246)(图 5～7), 其中 9
个为具有新颖骨架的化合物 $(117,118,142 \sim 144,157$, 158, 213, 246) (2008 2020 年), 73 个化合物具有抗菌、 细胞毒、杀虫、 $\alpha$-葡萄糖苷酶抑制活性、结核分枝杆菌 磷酸酶等生物活性(表 $5 \sim 7$ ).

从红树植物尖瓣海莲 (B. sexangula var. rhyn chopetala)内生真菌 Penicillium sp. TGM112 的次级代 谢产物中分离出了 8 个新的异香豆素类化合物 peniciisocoumarins $\mathrm{A} \sim \mathrm{H}(\mathbf{1 0 7} \sim \mathbf{1 1 4})^{[28]}$, 其中化合物 107, 111，112 均对棉铃虫 (Helicoverpa armigera Hubner) 有一定的生长抑制活性, $\mathrm{IC}_{50}$ 值均为 $200 \mu \mathrm{g} / \mathrm{mL}$. 从红树 植物拉关木 (L. racemose) 的内生真菌 P. coffeae MA-314 中分离到了 2 个新的异香豆素衍生物 penicoffrazins B, C $(\mathbf{1 1 5}, \mathbf{1 1 6})^{[76]}$. 从红树植物秋茄 $(K$. candel) 的内生真菌 P. commune QQF-3 的发酵提取物中 分离到了 10 个新的异香豆素类化合物 penisiocoumarins $\mathrm{A} \sim \mathrm{J}(\mathbf{1 1 7} \sim 126)^{[20]}$, 化合物 117 和 118 为一对 结构新颖的在 C-9/9'和 C-10/10'之间形成四元环的异香 豆素二聚体, 此类二聚体为首次从红树来源青霉属真菌 中分离得到; 活性测试发现化合物 119, 123, 125, 126 均 对 $\alpha$-葡萄糖苷酶具有抑制活性，其 $\mathrm{IC}_{50}$ 值分别为 (38.1 \pm 1.0$),(110.3 \pm 1.0),(78.1 \pm 1.0),(45.1 \pm 1.0)$ $\mu \mathrm{mol} / \mathrm{L}$, 构效关系研究表明 C-10/C-11 之间的环氧环为 关键的活性基团. 化合物 124 对结核分枝杆菌磷酸酶 (MptpB) 也有抑制作用, $\mathrm{IC}_{50}$ 值为 $20.7 \mu \mathrm{mol} / \mathrm{L}$. 从红树植 物尖瓣海莲 (B. sexangula var. rhynchopetala) 的内生真菌 P. citrinum HL-5126 的代谢产物中分离出了 3 个新的 二氢异香豆素 penicimarins $\mathrm{G} \sim \mathrm{I}(\mathbf{1 2 7} \sim \mathbf{1 2 9})^{[30]}$, 化合 物 127 和 128 均对表皮葡萄球菌(Staphylococcus epidermidis $) 、$ 金黄色葡萄球菌(S. aureus)、大肠杆菌 $(E$. coli)、蜡样芽胞杆菌(Bacillus cereus) 和溶藻弧菌(Vibrio alginolyticus) 具有抑制活性, 其 $\mathrm{IC}_{50}$ 值均为 $20 \mu \mathrm{mol} / \mathrm{L}$. 从红树植物尖瓣海莲 (B. Sexangula var. rhynchopetala) 的 
<smiles>COC(=O)CC[C@H]1Cc2ccc(O)c(OC)c2C(=O)O[C@H]1CCC(=O)OC</smiles><smiles>COC(=O)C1C(C(=O)OC)[C@H](C(=O)OC)[C@H]1c1cc2cc(OC)cc(O)c2c(=O)o1</smiles><smiles>COc1cc(O)c2c(=O)oc(CC3OC3C(=O)Oc3cc(O)c4c(=O)oc(CC(O)CCl)cc4c3)cc2c1</smiles><smiles>COc1cc(O)c2c(=O)oc(CC(Cl)CO)cc2c1</smiles><smiles>COc1cc(O)c2c(=O)oc(CC(Cl)CO)cc2c1O</smiles><smiles>CC(O)Cc1cc2c(O)c(O)cc(O)c2c(=O)o1</smiles>

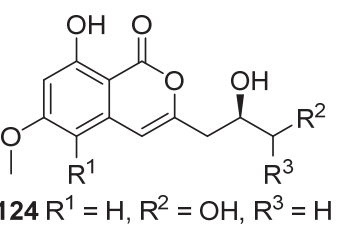<smiles>COc1ccc(O)c2c1C(=O)OC(CCCC(C)O)C2</smiles><smiles>COc1ccc(O)c2c1C(=O)OC(CCCC(C)=O)C2</smiles><smiles>CCOC(=O)CCC1Cc2cccc(O)c2C(=O)O1</smiles>

123 $125 \mathrm{R}^{1}=\mathrm{OH}, \mathrm{R}^{2}=\mathrm{OH}, \mathrm{R}^{3}=\mathrm{H}$<smiles>[R]c1c(O)cc2c(c1O)C(=O)OC(CCCCC)C2</smiles>

$130 \mathrm{R}=\mathrm{CH}_{3}$ $131 \mathrm{R}=\mathrm{H}$<smiles>CCC/C=C/[C@H]1Cc2cc(O)c(C)c(O)c2C(=O)O1</smiles><smiles>Cc1c(O)cc2c(c1O)C(=O)O[C@@H](C)[C@H]2C</smiles>

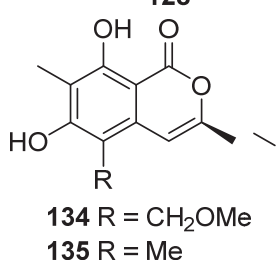<smiles>CCC(=O)OCC(Cl)Cc1cc2cc(OC)cc(O)c2c(=O)o1</smiles>

136

图 5 红树来源青霉属真菌产生的聚酮类化合物 107 136

Figure 5 Polyketides 107 $\sim \mathbf{1 3 6}$ produced by mangrove-derived Penicillum $\mathrm{sp}$.

表 5 新萜类化合物 $107 \sim 136$ 的生物活性与产生菌的来源 $a$

Table 5 Biological activities of new terpenoids 107 $\sim 136$ and the resources of producing strains

\begin{tabular}{|c|c|c|c|c|}
\hline Compound & Source & Bioactivity (MIC, $\left.\mathrm{LD}_{50}, \mathrm{IC}_{50}\right)$ & Region & Year \\
\hline $107 \sim 114$ & B. sexangula var. rhynchopetala & Helicoverpa armigera Hubner: $200 \mu \mathrm{g} / \mathrm{mL}(\mathbf{1 0 7}, \mathbf{1 1 1}, \mathbf{1 1 2})$ & Hainan & $2019^{[28]}$ \\
\hline 115,116 & Leaves of L. racemose & 1 & Hainan & $2019^{[76]}$ \\
\hline $117 \sim 126$ & Fruit of $K$. candel & $\begin{array}{l}\alpha \text {-glucosidase: } 38.1 \sim 110.3 \mu \mathrm{mol} / \mathrm{L}(\mathbf{1 1 9}, \mathbf{1 2 3}, \mathbf{1 2 5}, \mathbf{1 2 6}) \text {; } \\
\text { MptpB: } 20.7 \mu \mathrm{mol} / \mathrm{L}(\mathbf{1 2 4})\end{array}$ & Guangdong & $2018^{[20]}$ \\
\hline $127 \sim 129$ & B. sexangula var. rhynchopetala & $\begin{array}{l}\text { Staphylococcus epidermidis/S. aureus/E. coli/Bacillus cereus/ } \\
\text { Vibrio alginolyticus: } 20 \mu \mathrm{mol} / \mathrm{L}(\mathbf{1 2 7 / 1 2 8})\end{array}$ & Hainan & $2016^{[34]}$ \\
\hline $130 \sim 132$ & B. sexangula var. rhynchopetala & $\begin{array}{l}\text { salina: } 7.7 \sim 18.6 \mu \mathrm{g} / \mathrm{mL}(\mathbf{1 3 0} \sim \mathbf{1 3 2}), \text { E. coli/P. aeruginosal } \\
\text { V. harveyi/C. gloeosprioides: } 4 \mu \mathrm{g} / \mathrm{mL}(\mathbf{1 3 0})\end{array}$ & Hainan & $2016^{[31]}$ \\
\hline 133 & Root of $B$. sexangular & $\mathrm{K} 562: 18.9 \mu \mathrm{g} / \mathrm{mL}$ & Hainan & $2009^{[85]}$ \\
\hline 134 & Stem of $H$. littoralis & $\alpha$-glucosidase: $309.6 \mu \mathrm{mol} / \mathrm{L}$ & Guangdong & $2020^{[81]}$ \\
\hline 135 & Stem of H. littoralis & $\alpha$-glucosidase: $237.4 \mu \mathrm{mol} / \mathrm{L}$ & Guangdong & $2020^{[81]}$ \\
\hline 136 & Stem of $H$. littoralis & $\alpha$-glucosidase: $100.6 \mu \mathrm{mol} / \mathrm{L}$ & Guangdong & $2020^{[81]}$ \\
\hline
\end{tabular}


<smiles>[Y16]C[Y1]([H])([H])COC(C)=O</smiles>

图 6 红树来源青霉属真菌产生的聚酮类化合物 $137 \sim 152$

Figure 6 Polyketides 137 152 produced by mangrove-derived Penicillium sp.

表 6 新聚酮类化合物 $137 \sim 152$ 的生物活性与产生菌的来源 ${ }^{a}$

Table 6 Biological activities of new polyketides 137 $\sim 152$ and the resources of producing strains

\begin{tabular}{|c|c|c|c|c|}
\hline Compound & Source & Bioactivity (MIC, $\left.\mathrm{LD}_{50}, \mathrm{IC}_{50}\right)$ & Region & Year \\
\hline 137 & Leaves of $A$. marina & l & Hainan & $2015^{[49]}$ \\
\hline 138 & Leaves of $A$. marina & / & Hainan & $2015^{[49]}$ \\
\hline 139 & Branch of C. manghas & $\alpha$-glucosidase: $9.5 \pm 1.2 \mu \mathrm{mol} / \mathrm{L}$ & Hainan & $2014^{[61]}$ \\
\hline 140 & Branch of C. manghas & 1 & Hainan & $2014^{[61]}$ \\
\hline 141 & Branch of C. manghas & $\alpha$-glucosidase: $8.0 \pm 1.5 \mu \mathrm{mol} / \mathrm{L}$ & Hainan & $2014^{[61]}$ \\
\hline $142 \sim 144$ & L. racemosa & $\begin{array}{l}\text { Du145/HeLa/Huh/MCF-7/NCI-H460/ } \\
\text { SGC-7901/SW1990: } 3.8 \sim 10 \mu \mathrm{mol} / \mathrm{L}\end{array}$ & Hainan & $2013^{[70]}$ \\
\hline 145 & Leaves of L. racemose & $\begin{array}{l}\text { Fusarium oxysporum, f. sp. momordicae nov.f./ } \\
\text { C. gleosporioides: } 5 \mu \mathrm{mol} / \mathrm{L}\end{array}$ & Hainan & $2019^{[76]}$ \\
\hline 146 & Rhizospheric soil of mangrove plant & 1 & Hainan & $2019^{[10]}$ \\
\hline 147,148 & Soil of $R$. stylosa & DPPH: $14 \sim 15 \mu \mathrm{mol} / \mathrm{L}$ & l & $2015^{[59]}$ \\
\hline 149 & Leaves of A. marina & MG-63: $26 \mu \mathrm{mol} / \mathrm{L}, \mathrm{Tca} 8113: 35 \mu \mathrm{mol} / \mathrm{L}$ & Fujian & $2014^{[48]}$ \\
\hline $150 \sim 152$ & B. sexangula var. rhynchopetala & S. aureus: $6.25 \mu \mathrm{g} / \mathrm{mL}(\mathbf{1 5 0})$, A. salina: $78.5 \mu \mathrm{g} / \mathrm{mL}$ (152) & Hainan & $2019^{[29]}$ \\
\hline
\end{tabular}

$a /:$ No activity.

内生真菌 P. simplicissimum MA-332 中分离鉴定出了 3 个新的二氢异香豆素衍生物 penicisimpins $\mathrm{A} \sim \mathrm{C}(\mathbf{1 3 0} \sim$ 132 $)^{[34]}$, 化合物 130 132 对卤虫(A. salina) 具有致死活 性, 其 $\mathrm{LD}_{50}$ 值分别为 7.7, 36.4, $18.6 \mu \mathrm{g} / \mathrm{mL}$; 化合物 130 对大肠杆菌 (E. coli)、假单胞菌 (Pseudomonas aeruginosa)、哈维氏弧菌( $V$. harveyi) 和炭疽菌( C. gloeosprioides) 具有一定的抑制活性, MIC 值均为 $4 \mu \mathrm{g} / \mathrm{mL}$, 初步构效关系研究表明 C-7 位的甲基和 C-11 位的双键 为抗菌活性基团. 从红树植物海莲(B. sexangula) 的内 生真菌 Penicillium sp. 091402 中分离到了 1 个新的异 香豆素 $\left(3 R^{*}, 4 S^{*}\right)-6,8$-二羟基-3,4,7-三甲基异香豆素 $(133)^{[85]}$, 该化合物对肿瘤细胞 K562 具有中等的生长抑 制活性, 其 $\mathrm{IC}_{50}$ 值为 $18.9 \mu \mathrm{g} / \mathrm{mL}$. 从红树植物银叶树 $(H$. littoralis)的内生真菌 Penicillium sp. YYSJ-3 中分离鉴 定到了 3 个新的异香豆素类化合物 $(134 \sim 136)^{[81]}$, 化 合物 134 136 均对 $\alpha$-葡萄糖苷酶具有抑制活性，其 $\mathrm{IC}_{50}$ 值分别为 $309.6,237.4,100.6 \mu \mathrm{mol} / \mathrm{L}$.

从红树植物白骨壤 (A. marina) 的内生真菌 $P$. brocae MA-192 培养液中分离鉴定出了 1 个新的间苯 二酚酸衍生物 13-hydroxydihydroresorcylide (137)和 1 个新的开环间苯二酚酸类似物 brocapyrone $\mathrm{A}$ (138) ${ }^{[50]}$. 从红树植物海芒果 (C. manghas) 的内生真菌 Penicillium sp. HN29-3B1 的培养物中分离出了 3 个新 的蚓抑素衍生物 6-demethylpenisimplicissin (139), 5-hydroxy-penisimplicissin (140) 和 2-epihydroxydihy drovermistatin $(141)^{[61]}$, 化合物 139 和 141 对均 $\alpha$-葡萄 
<smiles>CC(C)CC1Oc2cccc(O)c2C(=O)CC1C1OC(=O)CC1C</smiles>

153<smiles>CC1=C2C(=O)c3c(O)cccc3OC2CC(C)C1O</smiles>

160

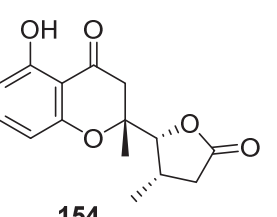

154<smiles>CC1C(=O)c2c(O)cccc2OC2=C1C(=O)c1c(O)cccc12</smiles>

161

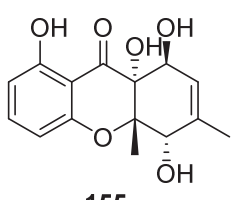

155

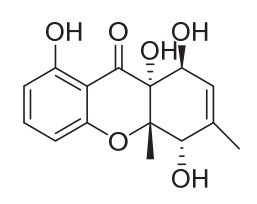

156

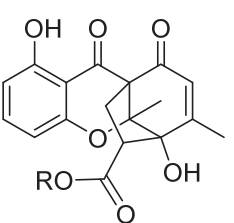

$157 \mathrm{R}=\mathrm{Et}$

$158 \mathrm{R}=\mathrm{Me}$

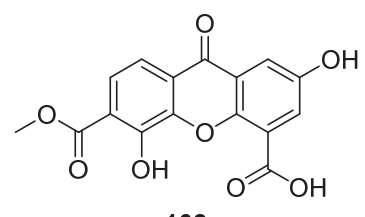

162

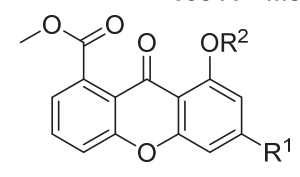

$163 \mathrm{R}^{1}=\mathrm{COOH}, \mathrm{R}^{2}=\mathrm{H}$ $164 \mathrm{R}^{1}=\mathrm{COOMe}, \mathrm{R}^{2}=\mathrm{Me}$

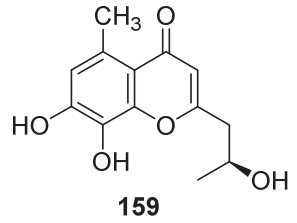

159

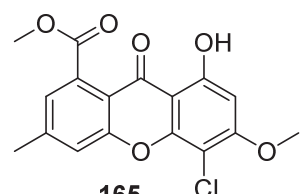

165

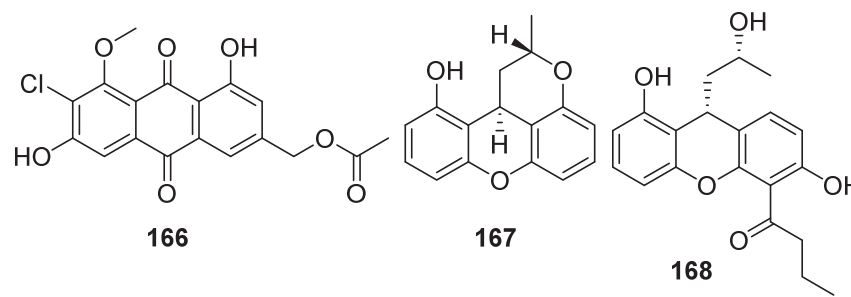

$\stackrel{\mathrm{OH}}{=}$

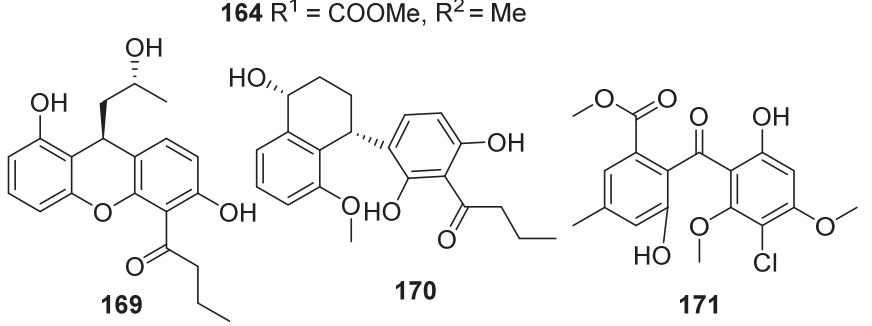

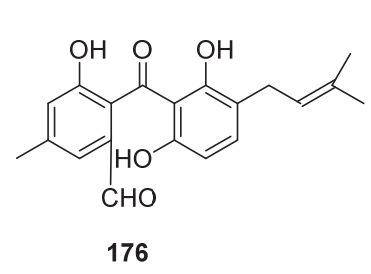

176

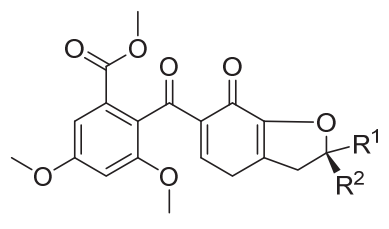

$177 R^{1}=H, R^{2}=M e$ $178 \mathrm{R}^{1}=\mathrm{Me}, \mathrm{R}^{2}=\mathrm{H}$

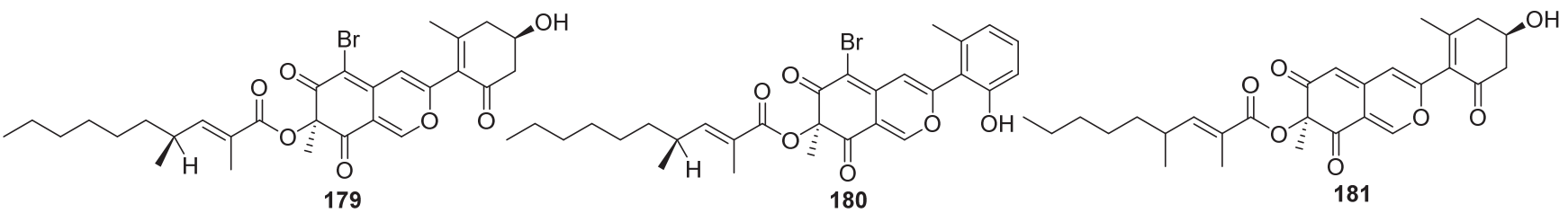

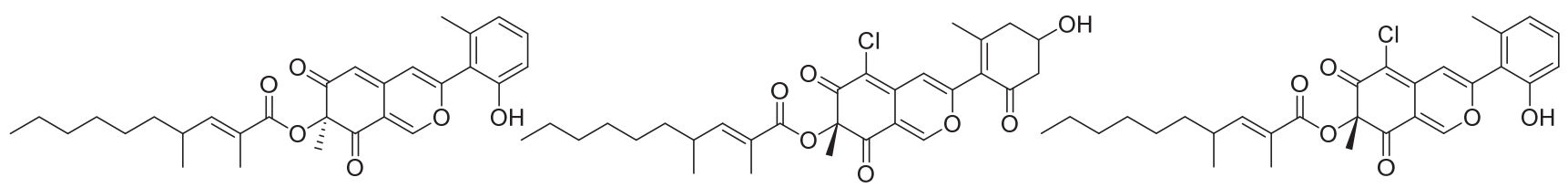

182

183

184

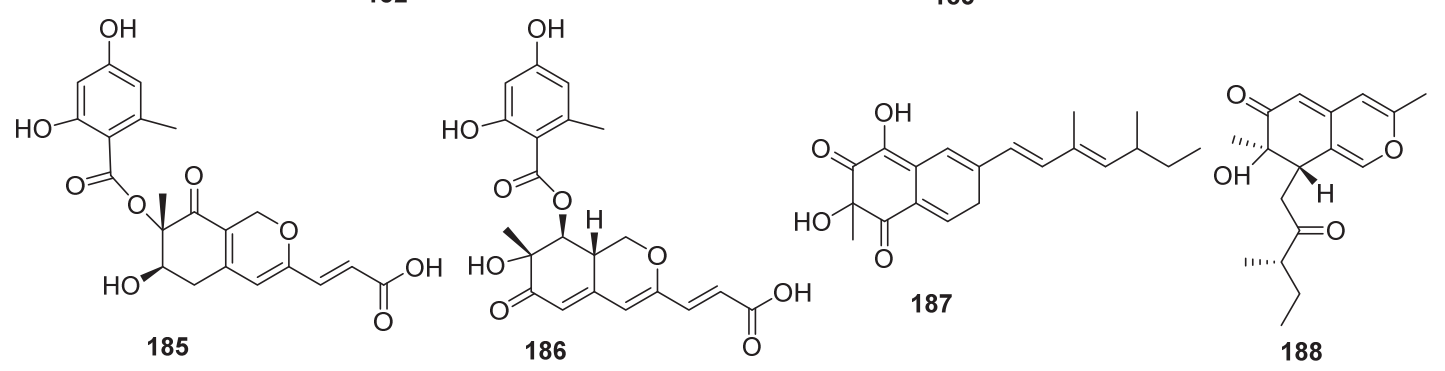<smiles>CCCC(=O)C1C(=O)OC2(C(=O)C=C3C=C(C)OC32)C1=O</smiles>

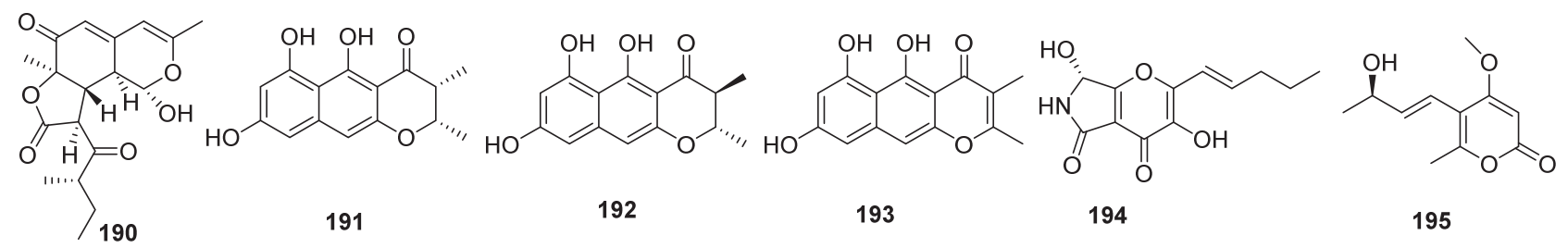



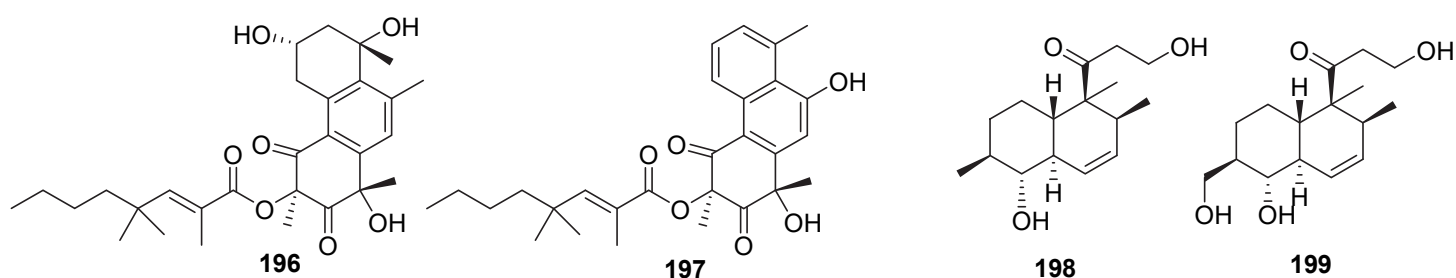

198

199

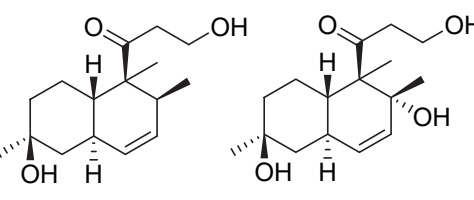

200

201<smiles>CC12C=CC3C(O)[C@@H](CO)CCC3[C@@]1(C)C(=O)CCO2</smiles>

202 (c)

203
204<smiles>CCC/C=C/C=C/C1CC(O)C(O)C(=O)C1CO</smiles>

205<smiles>CC1=CC(=O)C(C)(O)[C@H](O)C1</smiles>

207<smiles>COC1=CCC(/C(O)=C2/C(=O)C(C)(O)C(=O)C=C2C)C(O)=C1</smiles>

208<smiles>CC(=O)CC1(O)C(=O)C(=O)c2c(O)c(O)cc(O)c2C1=O</smiles><smiles>CO[C@]12CCCC[C@]1(C)Oc1cc(O)c(C)c3c1[C@@H](C)[C@@H](C)O[C@]32O</smiles>

210<smiles>Cc1c(O)cc(Oc2c(O)c(O)c(C)c3c2C(=O)O[C@H](C)C3C)c2c1C(C)[C@@H](C)O2</smiles>

211<smiles>COC1CCc2oc(=O)c3c(O)cc(C)cc3c21</smiles>

212<smiles>COC1=C(Cl)C(C)(O)c2c(oc(=O)c3c(O)cc(OC)cc23)[C@]12CC(O)C(=O)O2</smiles>

213<smiles>COC1=Cc2cc3c(=O)oc2=C(C1=O)C(O)(O)C(OC)=CC=3O</smiles>

209<smiles>COC1=C(Cl)C(C)(O)c2c(oc(=O)c3c(O)cc(OC)cc23)C1=O</smiles>

215<smiles>COC1=C(Cl)Cc2c(oc(=O)c3c(O)cc(OC)cc23)C1O</smiles>

216<smiles>CCCC[C@H]1C(=O)c2c(O)cc(O)cc2C[C@H]1CC(=O)/C=C/c1ccccc1</smiles>

217<smiles>CC(C)[C@H](C)/C=C/[C@H](C)[C@H]1C[C@@H](O)C2=C3C(=O)C=C4C=CCC[C@]4(C)[C@H]3CC[C@]21C</smiles><smiles>[R]CCC(/C=C/C=C(\C)C[C@H](C)/C=C/C=C/[C@H]1O[C@@H](C(=O)OC)[C@H]2C[C@@]1(C)O2)C[R]</smiles><smiles>CCCC(C)/C=C/C=C(\C)C[C@H](C)/C=C/C=C/C1OC(C)C=C[C@]1(C)O</smiles><smiles>[R]c1cc2c3c(c1[R])C(=O)O[C@]3(C)c1c(oc([R])c([R])c1=O)O2</smiles>
$223 \mathrm{R}^{1}=\mathrm{OH}, \mathrm{R}^{2}=\mathrm{R}^{3}=\mathrm{H}, \mathrm{R}^{4}={ }^{n} \mathrm{Pr}$ $224 \mathrm{R}^{1}=\mathrm{OH}, \mathrm{R}^{2}=\mathrm{H}, \mathrm{R}^{3}=\mathrm{OMe}, \mathrm{R}^{4}={ }^{n} \mathrm{Pr}$ $225 \mathrm{R}^{1}=\mathrm{OH}, \mathrm{R}^{2}=\mathrm{OMe}, \mathrm{R}^{3}=\mathrm{H}, \mathrm{R}^{4}=$ Propenyl<smiles>CC(C)=CCc1c2occc2c(C)c2ccc(=O)oc12</smiles>

226<smiles>CCCCc1c(C)c(C)cc(C(C)=O)c1O</smiles>

231

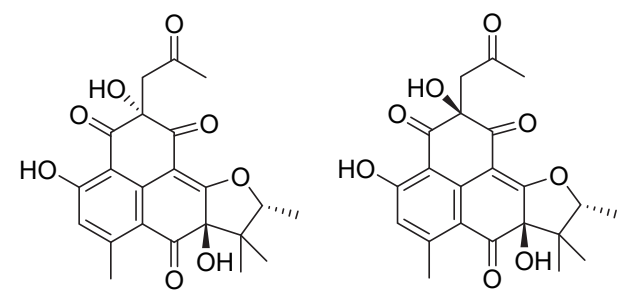

228<smiles>COC(C)C(C)(C)c1c(O)c2c3c(O)c1C(=O)c1c(O)cc(C)c-2c1C(O)(CC(C)=O)C3=O</smiles>

229<smiles>CC(=O)CC1(O)C(=O)C2=C(O)C=C(C)c3c4c(c(O)c2c31)C1(C)[C@H](C)O[C@H]4C1(C)C</smiles><smiles>CC(=O)c1cc(C)c(O)c(CC2=C(O)C(C)OC2=O)c1O</smiles>

233<smiles>CC1=C(c2cc(O)cc(O)c2)C(=O)[C@@H](O)C1</smiles>

234<smiles>COc1ccc(/C=C2\Oc3c(OC)c(O)cc(OC4OC(O)C(O)C(O)C(O)C4O)c3C2=O)cc1</smiles> 
<smiles>[Z16]COc1cccc2c1[C@H](O)C[C@@H](C)O2</smiles>

图 7 红树来源青霉属真菌产生的聚酮类化合物 $\mathbf{1 5 3} \sim \mathbf{2 4 6}$

Figure 7 Polyketides 153 246 produced by mangrove-derived Penicillium. sp

表 7 新聚酮类化合物 $153 \sim 246$ 的生物活性与产生菌的来源 $a$

Table 7 Biological activities of new polyketides $\mathbf{1 5 3} \sim \mathbf{2 4 6}$ and the resources of producing strains

\begin{tabular}{|c|c|c|c|c|}
\hline Compound & Source & Bioactivity (MIC, LD $50, \mathrm{IC}_{50}$ ) & Region & Year \\
\hline $153 \sim 156$ & Rhizospheric soil of mangrove plant & K562/MCF-7/Huh-7: 55.2 $67.5 \mu \mathrm{mol} / \mathrm{L}$ & Hainan & $2019^{[10}$ \\
\hline 157,158 & Rhizospheric soil of mangrove plant & 1 & Hainan & $2018^{[9]}$ \\
\hline 159 & Leaves of $K$. candel & Salmonell: $2.00 \pm 0.02 \mu \mathrm{mol} / \mathrm{L}$ & Guangdong & $2017^{[21}$ \\
\hline 160,161 & Rhizospheric soil of mangrove plant & H1975/MCF-7/K562/HL7702: 30 بmol/L (160) & Hainan & $2017^{[8]}$ \\
\hline 162 & Soil of $A$. ilicifolius & HeLa/Bel-7402/HEK-293/HCT-116/A549: 10.0 $30.6 \mu \mathrm{mol} / \mathrm{L}$ & Hainan & $2015^{[53}$ \\
\hline 163 & Stem of A. ilicifolius & 1 & Guangdong & $2008^{[55}$ \\
\hline 164 & Stem of A. ilicifolius & Fusarium oxysporumf. sp. Cubense: $12.5 \mu \mathrm{g} / \mathrm{mL}$ & Guangdong & $2008^{[55}$ \\
\hline 165 & B. sexangula var. rhynchopetala & 1 & Hainan & $2017^{[31}$ \\
\hline 166 & B. sexangula var. rhynchopetala & V. parahaemolyticus: $10 \mu \mathrm{mol} / \mathrm{L}$ & Hainan & $2017^{[31}$ \\
\hline $167 \sim 170$ & Stem of C. tagal & $\begin{array}{l}\text { Helicoverpa armigera Hubner: } 100 \sim 200 \mu \mathrm{g} / \mathrm{mL}(\mathbf{1 6 8} / \mathbf{1 6 9}) \\
\text { Culex quinquefasciatus: } 8.5 \sim 20.5 \mu \mathrm{g} / \mathrm{mL} \text { (except 168) }\end{array}$ & Nanhai & $2019^{[69}$ \\
\hline 171,172 & B. sexangula var. rhynchopetala & S. aureus: $20 \mu \mathrm{g} / \mathrm{mL}$ (171); A549: $15.7 \mu \mathrm{g} / \mathrm{mL}$ (172) & Hainan & $2019^{[32}$ \\
\hline 173 & Leaves of $S$. apetala & $\begin{array}{l}\text { Con A-induced (T cell): } 8.1 \mu \mathrm{g} / \mathrm{mL} \text {; } \\
\text { LPS-induced (B cell): } 9.3 \mu \mathrm{g} / \mathrm{mL}\end{array}$ & Guangdong & $2016^{[77}$ \\
\hline 174 & Leaves of $S$. apetala & 1 & Guangdong & $2016^{[77}$ \\
\hline 175 & B. gymnorrhiza & Aeromonas hydrophilia: $8 \mu \mathrm{g} / \mathrm{mL}$; A. salina: $25.3 \mu \mathrm{mol} / \mathrm{L}$ & Hainan & $2014^{[42}$ \\
\hline 176 & corniculatum & 1 & Fujian & $2008^{[62}$ \\
\hline 177,178 & Rhizospheric soil of mangrove plant & A. salina: $14.2 \sim 25.3 \mu \mathrm{mol} / \mathrm{L}$ & Techeng Isle & $2019^{[11]}$ \\
\hline 179,180 & Rhizospheric soil of mangrove plant & S. aureus/ Enterococcus faecalis: $3.13 \sim 12.5 \mu \mathrm{mol} / \mathrm{L}(\mathbf{1 8 0})$ & Hainan & $2019^{[4]}$ \\
\hline $181 \sim 184$ & Rhizospheric soil of mangrove plant & S. aureus: $3.13 \sim 6.25 \mu \mathrm{mol} / \mathrm{L}$ (except 181) & Hainan & $2017^{[6]}$ \\
\hline 185,186 & Branch of C. manghas & $\alpha$-glucosidase: $28.0 \mu \mathrm{mol} / \mathrm{L}$ & Hainan & $2014^{[60}$ \\
\hline 187 & B. sexangula & MDA-MB-435/HepG2/HCT-116/A549: $11.09 \sim 24.62 \mu \mathrm{mol} / \mathrm{L}$ & Guangdong & $2014^{[86}$ \\
\hline 188,190 & Stem of $K$. candel & $\alpha$-glucosidase: $24.5 \mu \mathrm{mol} / \mathrm{L}$ & Guangdong & $2011^{[20}$ \\
\hline $191 \sim 193$ & Rhizospheric soil of mangrove plant & $\begin{array}{l}\text { S. aureus (ATCC 43300, 33591, } 29213 \text { 25923): 12.5 50 } \\
\mu \mathrm{g} / \mathrm{mL}\end{array}$ & Hainan & $2019^{[3]}$ \\
\hline 194 & A. marina & S. aureus/V. harveyi/V. parahaemolyticus: $0.5 \mu \mathrm{g} / \mathrm{mL}$ & Hainan & $2015^{[43}$ \\
\hline 195 & Rhizospheric soil of mangrove & 1 & Hainan & $2018^{[15}$ \\
\hline 196, 197 & Rhizospheric soil of mangrove plant & S. aureus ATCC 33591: $3.13 \mu \mathrm{g} / \mathrm{mL}$ (196) & Hainan & $2019^{[4]}$ \\
\hline $198 \sim 203$ & Stem of $H$. tiliaceus & 1 & Guangdong & $2015^{[78}$ \\
\hline 204 & Root of $K$. candel & AchE: $12 \mu \mathrm{mol} / \mathrm{L}$ & Guangxi & $2014^{[25}$ \\
\hline 205 & Root of $K$. candel & AchE: $0.079 \mu \mathrm{mol} / \mathrm{L}$ & Guangxi & $2014^{[25}$ \\
\hline 206 & Root of $K$. candel & 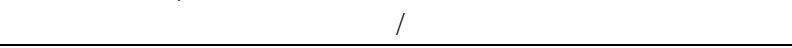 & Guangxi & $2014[25$ \\
\hline
\end{tabular}




\begin{tabular}{|c|c|c|c|c|}
\hline Compound & d Source & Bioactivity (MIC, LD $50, \mathrm{IC}_{50}$ ) & Region & Year \\
\hline 207 & Stem of $A$. corniculatum & A549: $1.45 \mu \mathrm{mol} / \mathrm{L}$ & Fujian & $2008^{[62]}$ \\
\hline 208 & Stem of $A$. corniculatum & P388: $1.38 \mu \mathrm{mol} / \mathrm{L}$ & Fujian & $2008^{[62]}$ \\
\hline 209 & Stem of $A$. corniculatum & 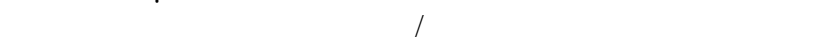 & Fujian & $2008^{[62]}$ \\
\hline $210 \sim 212$ & A. marina & HeLa/BEL-7402/HEK-293/HCT-116/A-549: 3.4 40.5 $\mu \mathrm{mol} / \mathrm{L}$ & 1 & $2015^{[52]}$ \\
\hline $213 \sim 216$ & Root of $H$. littoralis & 1 & Thailand & $2015^{[76]}$ \\
\hline 217 & A. ilicifolius & corticosterone-damaged $\mathrm{PC} 12$ cells & Hainan & $2014^{[57]}$ \\
\hline 218 & A. ilicifolius & U251/BT-325/SHG-44: $2.3 \sim 4.1 \mu \mathrm{mol} / \mathrm{L}$ & Hainan & $2014^{[57]}$ \\
\hline $219 \sim 221$ & B. sexangula & $\begin{array}{l}\mathrm{HeLa} / \mathrm{K} 562 / \mathrm{HCT}-116 / \mathrm{HL}-60 / \mathrm{A} 549 / \mathrm{MCF}-7: \\
1.2 \sim 9.8 \mu \mathrm{mol} / \mathrm{L}(\mathbf{2 1 9})\end{array}$ & Fujian & $2017^{[87]}$ \\
\hline 222 & B. sexangula & / & Fujian & $2017^{[87]}$ \\
\hline $223 \sim 225$ & Rhizospheric soil of mangrove plant & l & Techeng Isle & $2019^{[11]}$ \\
\hline 226 & A. marina & $\begin{array}{l}\mathrm{KB} / \mathrm{KBV} 200 / \mathrm{HeLa} / \\
\mathrm{HepG} / \mathrm{SGC} 7901: 5 \sim 42 \mu \mathrm{g} / \mathrm{mL}\end{array}$ & Hainan & $2012^{[51]}$ \\
\hline 227,228 & B. sexangula & 1 & Hainan & $2020^{[17]}$ \\
\hline 229 & Rhizospheric soil of mangrove plant & / & Hainan & $2020^{[17]}$ \\
\hline 230 & Rhizospheric soil of mangrove plant & / & Hainan & $2020^{[17]}$ \\
\hline $231 \sim 233$ & Stem of $A$. ilicifolius & MptpB: $0.16 \sim 1.375 \mu \mathrm{mol} / \mathrm{L}(\mathbf{2 3 1} / \mathbf{2 3 2})$ & Hainan & $2014^{[54]}$ \\
\hline 234 & Branch of C. manghas & 1 & Hainan & $2015^{[60]}$ \\
\hline 235 & A. marina & / & Fujian & $2015^{[52]}$ \\
\hline 236 & A. marina & / & Fujian & $2015^{[52]}$ \\
\hline 237 & Leaves of $S$. apetala & / & Hainan & $2012^{[77]}$ \\
\hline 238 & Soil of $B$. gymnorrhiza & / & Fujian & $2012^{[41]}$ \\
\hline 239 & Rhizospheric soil of mangrove plant & 1 & Fujian & $2020^{[18]}$ \\
\hline 240 & K. candel & AChE/ $\alpha$-glucosidase /Antioxidant: $>100 \mu \mathrm{mol} / \mathrm{L}$ & Hainan & $2020^{[27]}$ \\
\hline 241 & B. sexangula var. rhynchopetala & 1 & Hainan & $2016^{[33]}$ \\
\hline 242 & Rhizospheric soil of mangrove plant & $\begin{array}{l}\text { Attenuate OGD-induced cell viability loss in } \\
\text { rat spinal cord astrocytes }\end{array}$ & Hainan & $2020^{[7]}$ \\
\hline 243,244 & Stem of ilicifolius & / & Hainan & $2014^{[54]}$ \\
\hline 245 & K. candel & / & Hong Kong & $2009^{[26]}$ \\
\hline 246 & K. candel & AChE/ $\alpha$-glucosidase/Antioxidant: $>100 \mu \mathrm{mol} / \mathrm{L}$ & Hainan & $2020^{[27]}$ \\
\hline
\end{tabular}

糖苷酶具有明显的抑制作用，其 $\mathrm{IC}_{50}$ 值分别为 $9.5 \pm 1.2$ 和 $8.0 \pm 1.5 \mu \mathrm{mol} / \mathrm{L}$. 弯孢菌素衍生物一般从 Curvularia sp., Aspergillus sp., Alternaria sp. 和 Penicillium sp. 真菌中 分离得到. 从红树植物榄李 (L. racemosa) 的内生真菌 $P$. sumatrense MA-92 的提取物中首次发现了 3 个硫代弯 孢菌素衍生物 sumalarins $\mathrm{A} \sim \mathrm{C}(\mathbf{1 4 2} \sim \mathbf{1 4 4})^{[70]}$, 目前尚 未从其他生境来源青霉属真菌中发现硫代弯狍菌素类 化合物, 可推测强酸等土壤环境对真菌的代谢途径产生 了影响. 化合物 142 144 对肿瘤细胞 Du145, HeLa, Huh, MCF-7, NCI-H460, SGC-7901 和 SW1990 表现出较 强的生长抑制毒性, 其 $\mathrm{IC}_{50}$ 值为 $3.8 \sim 10 \mu \mathrm{mol} / \mathrm{L}$. 从红 树植物拉关木 (L. racemose) 的内生真菌 P. coffeae MA-314 中分离到了 1 个含有 $\delta$-内酯片段的新化合物 penicoffeazine A (145) ${ }^{[76]}$, 该化合物对尖孢镰刀菌 (Fusarium oxysporum f. sp. momordicae nov. f.) 和炭疽病 菌(C. gleosporioides) 显示较强的抗菌活性, MIC 值均为 $5 \mu \mathrm{mol} / \mathrm{L}$. 从红树林沉积物来源的真菌 Penicillium sp. SCSIO041218 中分离到了 1 个新的大环双内酯 mangrovlide A (146) ${ }^{[10]}$. 从红树植物红海榄 $(R$. stylosa) 的内生真菌 P. solitum GWQ-143 的提取液中分离得到 了 2 个新的化合物 penipyrols A, B $(\mathbf{1 4 7}, \mathbf{1 4 8})^{[59]}$, 化合 物 147, 148 均对 DPPH 自由基具有较强的清除作用, 其 $\mathrm{IC}_{50}$ 值分别为 15 和 $14 \mu \mathrm{mol} / \mathrm{L}$. 从红树植物白骨壤 $(A$. marina)内生真菌 Penicillium sp. FJ-1 中分离到了 1 个新 的螺环化合物 15-hydroxy- $6 \alpha, 12$-epoxy-7 $\beta, 10 \alpha, 11 \beta H$ spiroax-4-ene-12-one (149) ${ }^{[48]}$, 该化合物对肿瘤细胞株 MG-63 和 Tca8113 显示一定的生长抑制活性, 其 $\mathrm{IC}_{50}$ 值 分别为 26 和 $35 \mu \mathrm{mol} / \mathrm{L}$. 目前仅从自然界中发现 17 个 6,7-二羟基辛-2,4-二烯类化合物，它们大多从药用植物 Goniothalamus giganteus 和 Litchi chinensis sonn, 真菌 Plectania sp.和 Aspergillus versicolor 中被分离得到[29], 其中 3 个新的 6,7-二羟基辛-2,4-二烯类化合物 penicilactones $\mathrm{A} \sim \mathrm{C}(\mathbf{1 5 0} \sim \mathbf{1 5 2})^{[29]}$ 从红树植物尖瓣海莲 (B. Sexangula var. rhynchopetala) 来源的青霉属内生真 菌 Penicillium sp. TGM112 中分离得到, 尚未从其他生 境来源的青霉属真菌中发现此类化合物. 化合物 $\mathbf{1 5 0}$ 对 
金黄色葡萄球菌( S. aureus) 有较强的抑制活性, 其 MIC 值为 $6.25 \mu \mathrm{g} / \mathrm{mL}$; 化合物 151 具有较好的卤虫(A. salina) 致死活性，其 $\mathrm{IC}_{50}$ 值为 $78.5 \mu \mathrm{g} / \mathrm{mL}$.

从红树林沉积物衍生的内生真菌 Penicillium sp. SCSIO041218 中分离到了 4 个新的色酮衍生物(153 156 $)^{[1]}$ 和 2 个骨架新颖的具有 $6 / 6 / 6 / 5$ 多环骨架的色酮 penixantones C, D $(\mathbf{1 5 7}, \mathbf{1 5 8})^{[10]}$, 通过电子圆二色谱 (ECD)测试发现化合物 157 为一对外消旋体, 化合物 157 和 158 的相对构型和绝对构型尚未确定; 化合物 157 和 158 均对肿瘤细胞 K562、MCF-7、Huh-7 表现出 较弱的细胞毒活性, 其 $\mathrm{IC}_{50}$ 值分别为 55.2 和 $56.5 \mu \mathrm{mol} / \mathrm{L}$ (K562)、61.1 和 $58.6 \mu \mathrm{mol} / \mathrm{L}$ (MCF-7)、67.5 和 $64.2 \mu \mathrm{mol} / \mathrm{L}$ (Huh-7). 从来源于红树植物秋茄 (K. candel) 叶片的真 菌 P. aculeatum No. 9EB 中分离出了 1 个新的色酮衍生 物 (2'S*)-2-(2'-hydroxy-propyl)-5-methyl-7,8-dihydroxychromone (159) ${ }^{[21]}$, 其对沙门氏菌(Salmonell) 有较强的 抑制活性, 其 MIC 值为 $2.00 \pm 0.02 \mu \mathrm{mol} / \mathrm{L}$.

从红树林土壤来源真菌 Penicillium sp. SYFz-1 的 提取物中分离出了 2 个新的氧杂葱酮衍生物 penixanthones A, B $(\mathbf{1 6 0}, \mathbf{1 6 1})^{[8]}$, 化合物 $\mathbf{1 6 0}$ 对人肿瘤细 胞 H1975, MCF-7, K562 及 HL7702 具有微弱的抑制活 性, 其 $\mathrm{IC}_{50}$ 值均为 $30 \mu \mathrm{mol} / \mathrm{L}$. 从红树植物老鼠簕 $(A$. ilicifolius) 的内生真菌 P. chrysogenum HND11-24 分离出 了 1 个新的氧杂蒽酮化合物(162 $)^{[53]}$, 化合物 $\mathbf{1 6 2}$ 对肿瘤 细胞 HeLa, Bel-7402, HEK-293, HCT-116 和 A549 表现出 显著的抑制活性，其 $\mathrm{IC}_{50}$ 值分别为 10.0, 30.6, 28.5, 19.0 和 $16.9 \mu \mathrm{mol} / \mathrm{L}$. 从红树植物老鼠簕 (A. ilicifolius) 的内生 真菌 Penicillium sp. (ZZF 32 ${ }^{\#}$ )培养液中分离得到 2 个氧 杂葱酮化合物 8-(methoxycarbonyl)-1-hydroxyl-9-oxo9H-xanthene-3-carboxylic acid (163), dimethyl-8methoxy-9-oxo-9H-xanthene-1,6-dicarboxylate $\quad(\mathbf{1 6 4})^{[55]}$, 化合物 164 对尖孢镰刀菌 (Fusarium oxysporumf. sp. cubense) 显示出显著的抗菌活性, MIC 值为 $12.5 \mu \mathrm{g} / \mathrm{mL}$. 从红树植物尖瓣海莲 (B. Sexangula var. rhynchopetala) 的 内生真菌 P. citrinum HL-5126 的乙酸乙酯提取物中分离 出了 2 个新化合物 4-chloro-1-hydroxy-3-methoxy-6- methyl-8-methoxy carbonyl-xanthen-9-one (165) 和 20acetoxy-7-chloro-citreorosein (166) ${ }^{[31]}$, 化合物 166 对副 溶血弧菌 $(V$. parahaemolyticus $)$ 具有较强的抑制活性, $\mathrm{MIC}$ 值为 $10 \mu \mathrm{mol} / \mathrm{L}$. 从红树植物角果木 (C. tagal) 的内 生真菌 Penicillium sp. JY246 中分离得到了 4 个新的氧 杂葱衍生物 penicixanthenes $\mathrm{A} \sim \mathrm{D}(\mathbf{1 6 7} \sim \mathbf{1 7 0})^{[69]}$, 化合 物 168 和 169 对棉铃虫幼虫 (Helicoverpa armigera Hubner) 的生长具有抑制活性, 其 $\mathrm{IC}_{50}$ 值分别为 100 和 $200 \mu \mathrm{g} / \mathrm{mL}$, 且化合物 $\mathbf{1 6 7 , 1 6 9}$ 和 170 均对致倦库蚊幼
虫(Culex quinquefasciatus) 具有致死活性, 其 $\mathrm{LC}_{50}$ 值 分别为 $8.5 \pm 1.16,11.6 \pm 0.58$ 和 $20.5 \pm 1 \mu \mathrm{g} / \mathrm{mL}$.

从红树植物尖瓣海莲 (B. Sexangula var. rhynchopetala) 的内生真菌 $P$. citrinum HL-5126 的乙酸乙酯 提取物中分离到了 2 个新的化合物 penibenzophenones

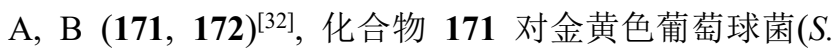
aureus) 具有一定的抑制活性, 其 MIC 值为 $20 \mu \mathrm{g} / \mathrm{mL}$; 化合物 172 对肿瘤细胞 A549 具有细胞毒活性, 其 $\mathrm{IC}_{50}$ 值为 $15.7 \mu \mathrm{g} / \mathrm{mL}$. 从红树植物无瓣海桑 (S. apetala) 的内 生真菌 Penicillium sp. ZJ-SY2 代谢产物中分离得到了 2 个新的二苯甲酮衍生物 peniphenone (173)和 methyl peniphenone (174) ${ }^{[77]}$, 化合物 173 对小鼠脾淋巴细胞的 Con A-诱导的 T 细胞和 LPS 诱导的 B 细胞增殖具有 较强的免疫抑制活性, 其 $\mathrm{IC}_{50}$ 值分别为 8.1 和 9.3 $\mu \mathrm{g} / \mathrm{mL}$. 从红树植物木榄 (B. gymnorrhiza) 的内生真菌 Penicillium sp. MA-37 中分离出了 1 个新的二苯甲酮类 化合物 iso-monodictyphenone (175) ${ }^{[42]}$, 且该化合物对 嗜水气单孢菌(Aeromonas hydrophilia) 具有抑制活性, 其 MIC 值为 $8 \mu \mathrm{g} / \mathrm{mL}$; 化合物 $\mathbf{1 7 5}$ 对卤虫(A. salina)显示 出一定的致死活性, 其 $\mathrm{LD}_{50}$ 值为 $25.3 \mu \mathrm{mol} / \mathrm{L}$. 从红树 植物桐花树 (A. corniculatum) 的内生真菌 Penicillium sp. JP-1 提取物中分离出了 1 个新的化合物 arugosin I $(\mathbf{1 7 6})^{[62]}$. 从红树林沉积物的内生真菌 P. pinophilum SCAU037 培养物中获得了 2 个新化合物 pinophilones A, $\mathrm{B}(\mathbf{1 7 7}, \mathbf{1 7 8})^{[12]}$.

嗜氮酮类化合物主要从海洋来源 Pleosporales sp., Hypoxylon sp., Chaetomium species 和 Diaporthe sp.等属 的真菌中分离得到, 目前已分离获得 430 余个此类化合 物，具有抗菌、抗病毒、抗氧化、细胞毒性和抗炎等生 物 [03-106]. 通过在培养基中添加溴化钠, 陈等从红树林 根际土壤真菌 P. janthinellum HK1-6 分离出了 2 个新的 溴代嗜氮酮类化合物 penicilones $\mathrm{G}, \mathrm{H}(\mathbf{1 7 9}, \mathbf{1 8 0})^{[4]}$ 和 4 个新的嗜氮酮 penicilones $A \sim D(181 \sim 184)^{[6]}$, 可推测 该菌能够利用溴化钠进行嗜氮酮类化合物的生物合成, 其中化合物 180 对金黄色葡萄球菌 (S. aureus ATCC 43300, 33591，2921 和 25923)、肠球菌(Enterococcus faecalis ATCC 51299)具有较好的抗菌活性, 其 $\mathrm{IC}_{50}$ 值分 别为 $12.5,3.13,6.25,12.5$ 和 $3.13 \mu \mathrm{mol} / \mathrm{L}$; 化合物 $\mathbf{1 8 2}$ 对金黄色葡萄球菌 (S. aureus ATCC 43300 和 33591) 显示 出强的抑制活性，其 $\mathrm{IC}_{50}$ 值均为 $3.13 \mu \mathrm{mol} / \mathrm{L}$; 化合物 183 和 184 对金黄色葡萄球菌(S. aureus ATCC 43300 和 33591)均显示出较强的抑制活性, 其 $\mathrm{IC}_{50}$ 值均为 6.25 $\mu \mathrm{mol} / \mathrm{L}$, 构效关系研究表明 7-O- $(E)-2,4-$ 二甲基葵烷-2烯酰基为重要抗菌活性基团. 从红树植物海芒果 $(C$. manghas)的内生真菌 Penicillium sp. HN29-3B1 的培养 
物中分离出了 2 个新的聚酮类化合物 pinazaphilones A, B $(\mathbf{1 8 5}, 186)^{[60]}$, 化合物 186 具有 $\alpha$-葡萄糖苷酶抑制活 性, 其 $\mathrm{IC}_{50}$ 值为 $28.0 \mu \mathrm{mol} / \mathrm{L}$. 从红树植物海莲 (B. sex-

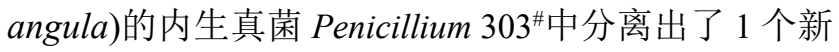

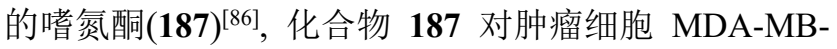
435, HepG2, HCT-116 和 A549 均表现出中等强度的生长 抑制活性，其 $\mathrm{IC}_{50}$ 值分别为 $24.62 \pm 2.89,17.92 \pm 1.86$, $11.09 \pm 1.41$ 和 $16.63 \pm 1.15 \mu \mathrm{mol} / \mathrm{L}$. 从红树植物秋茄 $(K$. candel) 的内生真菌 P. chermesinum ZH4-E2 培养物中分 离出了 3 个新的嗜氮酮 chermesinones $\mathrm{A} \sim \mathrm{C}(\mathbf{1 8 8} \sim$ 190 $)^{[22]}$, 其中化合物 188 对 $\alpha$-葡萄糖苷酶有较弱的抑 制作用, $\mathrm{IC}_{50}$ 值为 $24.5 \mu \mathrm{mol} / \mathrm{L}$. 从红树林根际土壤来 源的内生真菌 Penicillium sp. HK1-22 中分离到了 3 个 新的荎- $\gamma$-吡喃酮化合物 peninaphones $\mathrm{A} \sim \mathrm{C}(191 \sim$ $193)^{[3]}$ ，化合物 191 193 对金黄色葡萄球菌 (S. aureus ATCC 43300, 33591, 29213 和 25923)均具有抑制活性, 其 MIC 值为 $12.5 \sim 50 \mu \mathrm{g} / \mathrm{mL}$. 从红树植物白骨壤 $(A$. marina) 的内生真菌 P. brocae MA-231 的提取物中分离 出了 1 个新的多氧二氢吡喃酮衍生物 pyranonigrin $\mathrm{F}$ $(194)^{[43]}$, 该化合物对金黄色葡萄球菌(S. aureus)、哈维 氏弧菌 ( . h harveyi) 和副溶血弧菌 $(V$. parahaemolyticus $)$ 均具有一定的抗菌活性, 其 MIC 值均为 $0.5 \mu \mathrm{g} / \mathrm{mL}$. 从 红树林根际土壤来源的内生真菌 P. camemberti OUCMDZ-1492 中分离得到了 1 个新的 $\alpha$-吡喃酮类化合 物(195) ${ }^{[15]}$.

从红树林根际土壤来源的真菌 P. janthinellum HK1-6 分离出了 2 个新的澳代三环聚酮 penijanthinones A，B $(196,197)^{[4]}$, 化合物 196 对金黄色葡萄球菌 $(S$. aureus ATCC 33591)表现出一定的抑制活性, MIC 值为 $3.13 \mu \mathrm{g} / \mathrm{mL}$, 构效关系研究表明 $\mathrm{Br}$ 为重要的活性基团. 从红树植物黄瑾 (H. tiliaceus $)$ 的内生真菌 P. auranti-

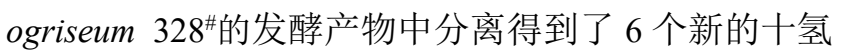
荎环聚酮化合物 peaurantiogriseols $\mathrm{A} \sim \mathrm{F}(\mathbf{1 9 8} \sim$ 203 $)^{[78]}$. 从红树植物秋茄 $(K$. candel $)$ 的内生真菌 Penicillium sp. sk14JW2P 中分离得到了 3 个聚酮 13-hydroxypalitantin (204), ( + )-palitantin (205) 和

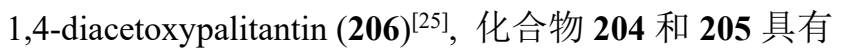
一定的乙酰胆碱酯酶(AchE) 抑制活性, 其 $\mathrm{IC}_{50}$ 值分别 为 12 和 $0.079 \mu \mathrm{mol} / \mathrm{L}$. 从红树植物桐花树 $(A$. corniculatum) 的内生真菌 Penicillium sp. JP-1 中分离到了 3 个聚 酮 leptosphaerone C (207), penicillenone (208) 和 9-demethyl FR-901235 (209) ${ }^{[62]}$, 化合物 209 对肿瘤细胞 A549 具有较强的细胞毒活性, 其 $\mathrm{IC}_{50}$ 值为 1.45 $\mu \mathrm{mol} / \mathrm{L}$; 化合物 208 对肿瘤细胞 P388 具有明显的细胞 毒活性, 其 $\mathrm{IC}_{50}$ 值为 $1.38 \mu \mathrm{mol} / \mathrm{L}$. 从红树植物老鼠簕
(A. ilicifolius)的内生真菌 P. chrysogenum HND 11-24 的 提取物中分离出了 3 个桔霉素类似物 penicitols $\mathrm{A} \sim \mathrm{C}$ $(210 \sim 212)^{[52]}$, 化合物 210 212 对 5 株肿瘤细胞( HeLa, BEL-7402, HEK-293, HCT-116, A-549)的生长均具有较 强的抑制作用，其 $\mathrm{IC}_{50}$ 值分别为 4.6 10.5，3.4 9.6、 $10.8 \sim 40.5 \mu \mathrm{mol} / \mathrm{L}$. 从红树植物银叶树 (H. littoralis) 的 内生真菌 P. chermesinum strain HLit- ROR2 中分离出 4 个新的化合物 $(213 \sim 216)^{[80]}$, 其中化合物 213 是骨架新 颖的具有独特螺环结构的 $\delta$-内酯环的四环聚酮类化 合物. 从红树植物老鼠簕 (A. ilicifolius) 的内生真菌 Penicillium sp. FJ-1 的提取物中分离出了 2 个新的化合 物(2R,3S)-pinobanksin-3-cinnamate (217) 和 15-hydroxy(22E,24R)-ergosta-35,8(14),22-tetraen-7-one (218) ${ }^{[57]}$, 其 中化合物 217 对皮质酮损伤的 $\mathrm{PC} 12$ 细胞具有较强的神 经保护作用, 化合物 218 对胶质瘤细胞(U251, BT-325, SHG-44) 具有较强的细胞毒性, 其 $\mathrm{IC}_{50}$ 值分别为 3.2, 4.1 和 $2.3 \mu \mathrm{mol} / \mathrm{L}$. 从深海来源的真菌 T. aculeatus 和红树林 来源的内生真菌 P. variabile 混合培养基中分离出了 4 个 新的聚酮化合物 penitalarins $\mathrm{A} \sim \mathrm{C}(\mathbf{2 1 9} \sim \mathbf{2 2 1})$ 和 nafuredin B (222) $)^{[87]}$, 化合物 219 221 为具有 3,6-二氧 杂环[3.1.0.1.0]环的聚酮类化合物; 化合物 219 对 6 种肿 瘤细胞 HeLa, K562, HCT-116, HL-60, A549 和 MCF-7 的 生长均有抑制作用，其 $\mathrm{IC}_{50}$ 值分别为 $5.5,2.9,1.4,1.2$, 5.1 和 $9.8 \mu \mathrm{mol} / \mathrm{L}$. 从红树林沉积物来源的内生真菌 $P$. pinophilum SCAU037 的培养物中获得 3 个新的 funicone 衍生物 pinophilones $\mathrm{C} \sim \mathrm{E}(\mathbf{2 2 3} \sim \mathbf{2 2 5})^{[12]}$. 从红树植物 白骨壤(A. marina)的内生真菌 Penicillium sp. ZH16 的 发酵提取物中分离出 1 个新的呋喃香豆素 5-methyl8-(3-methylbut-2-enyl) furanocoumarin (226 ${ }^{[51]}$, 该化合 物对 KB, KBV200, HeLa, HepG2 和 SGC7901 细胞均具 有较强的生长抑制活性，其 $\mathrm{IC}_{50}$ 值分别为 $5,10,29,21$ 和 $42 \mu \mathrm{g} / \mathrm{mL}$. 从红树林根际土壤来源的内生真菌 $P$. herquei MA-370 的培养物中分离到了 4 个新化合物 aceneoherqueinones A, B (227, 228), (+)-aceatrovenetinone A (229)和 $(+)$-aceatrovenetinone B (230) ${ }^{[17]}$. 通过 分子对接模拟其活性结合位点, 化合物 227 230 可作 为潜在的候选化合物或结构优化的起始化合物, 用于开 发治疗高血压的新药. 从红树植物老鼠簕(A. ilicifolius) 的内生真菌 P. dipodomyicola HN4-3A 中分离到 3 个新的 化合物 peniphenones $\mathrm{B} \sim \mathrm{D}(\mathbf{2 3 1} \sim \mathbf{2 3 3})^{[54]}$, 化合物 231 和 232 对结核分枝杆菌磷酸酶(MptpB) 有较强的抑制 活性, 其 $\mathrm{IC}_{50}$ 值分别为 $0.16 \pm 0.02$ 和 $1.37 \pm 0.05 \mu \mathrm{mol} / \mathrm{L}$. 从红树植物海芒果 (C. manghas) 的内生真菌 Penicillium sp. HN29-3B1 中分离得到了 1 个新的酚类化合物 $(\mathbf{2 3 4})^{[60]}$. 从红树植物木榄 (B. gymnorrhiza) 的内生真菌 
P. citrinum 分离到了 2 个新化合物(Z)-7,4'-dimethoxy6-hydroxyaurone-4- $O-\beta$-glucopyranoside (235) 和 ( - )-4$O-\left(4-O-\beta\right.$-D-glucopyranosylcaffeoyl) quinic acid $(\mathbf{2 3 6})^{[52]}$, 化合物 236 对鼻咽癌 $\mathrm{KB}$ 细胞表现出较强的细胞毒活 性, 其 $\mathrm{IC}_{50}$ 值为 $10 \mu \mathrm{mol} / \mathrm{L}$. 从红树植物木榄 $(B . g y m-$ norrhiza) 的内生真菌 Penicillium sp. MA-37 的代谢产物 中分离出了 1 个新的二苯醚衍生物 $\Delta^{1^{\prime}, 3^{\prime}}-1^{\prime}$-dehydroxypenicillide $(237)^{[41]}$. 从红树林沉积物来源的真菌 P. pinophilum H608 分离出了 1 个新的代谢产物 5'-hydroxypenicillide $(\mathbf{2 3 8})^{[13]}$. 从红树林沉积物来源的内生真菌 P. polonicum $\mathrm{H} 175$ 中分离到了 2 个新化合物 6-acetyl-4methoxy-3,5-dimethyl-2H-pyran-2-one (239) ${ }^{[18]}$. 从红树 植物秋茄 (K. candel) 的内生真菌 P. citrinum QJF-22 的 培养物中分离得到 1 个新的化合物 $(2 R, 4 S)$-5-methoxy2-methyl-3,4-dihydro-2H-1-benzopyran-4-ol (240 $)^{[27]}$ ，其 中化合物 240 具有较弱的抗氧化、乙酰胆碱酷酶(AchE) 和 $\alpha$-葡萄糖苷酶活性, $\mathrm{IC}_{50}$ 值都超过 $100 \mu \mathrm{mol} / \mathrm{L}$. 从红 树植物尖瓣海莲 (B. Sexangula var. rhynchopetala) 的内 生真菌 P. citrinum HL-5126 的培养液中分离得到了 1 个 新的苯丙吡喃酮衍生物 $\left(2 R^{*}, 4 R^{*}\right)-3,4-$ dihydro-5methoxy-2-methyl-2H-1-benzopyran-4-ol (241) ${ }^{[33]}$. 从红 树植物根际土壤的内生真菌 P. janthinellum HK1-6 的培 养液中分出了 1 个新的化合物 $(\mathbf{2 4 2})^{[7]}$. 且用噻唑蓝 (MTT)法测定化合物 242 的神经保护活性. 且该化合物 在 $50 \mathrm{mg} / \mathrm{mL}$ 浓度下对四种食源性致病菌(副伤寒沙门氏 菌(Salmonell paratyphi)、大肠杆菌(E. coli)、志贺氏菌 (Shigella) 和副溶血弧菌( V. parahaemolyticus $)$ )均具有 显著的抑制活性. 从红树植物老鼠簕(A. ilicifolius) 的内 生真菌 P. dipodomyicola HN4-3A 中分离到了 1 个新的 含有苯环的 6,6-螺环酮对映体 $(\mathbf{2 4 3}, \mathbf{2 4 4})^{[54]}$. 从红树植 物秋茄 (K. candel) 的内生真菌 Penicillium sp. (SBE-8)的 发酵提取物中分离到了 1 个新的代谢产物 7-hydroxyjanthinone (245) ${ }^{[26]}$. 从红树植物秋茄 $(K$. can$d e l$ ) 的内生真菌 P. citrinum QJF-22 培养物中分离得到 了 1 个新的化合物( $\left.2 S, 4 R, 2^{\prime} S, 4^{\prime} R\right)-4,4^{\prime}$-oxybis(5-methoxy2-3,4-dihydro-2H-1-benzopyran) (246) ${ }^{[27]}$.

\section{5 其他类化合物}

已从红树来源青霉属内生真菌的代谢产物中分离 鉴定出其他类化合物 30 个(247～276) (2010～2020 年)(图 8), 其中 15 个化合物具有抗菌、抗氧化、细胞毒 及杀虫等生物活性(表 8 ).

从红树林植物白骨壤 (A. marina) 的内生真菌 Penicillium sp. FJ-1 提取物中分离了 1 个新化合物 4-(20,30-dihydroxy-30-methyl-butanoxy)-phenethanol $(\mathbf{2 4 7})^{[48]}$, 该化合物对 Tca8113 和 MG-63 细胞具有一定
的增殖抑制活性，其 $\mathrm{IC}_{50}$ 值分别为 2.0 和 $35 \mu \mathrm{mol} / \mathrm{L}$. 从 红树植物海芒果(C. manghas)的内生真菌 Penicillium sp. HN29-3B1 培养物中分离得到了 1 个新的酚类化合物 $(\mathbf{2 4 8})^{[60]}$. 从红树植物海漆 (E. agallocha) 的内生真菌 P. expansum 091006 培养物中分离出了 4 个含二苯醚单元

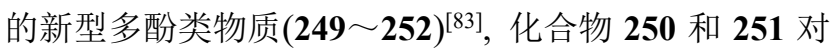
肿瘤细胞 HL-60 均表现出较弱的细胞毒活性, 其 $\mathrm{IC}_{50}$ 值 分别为 18.2 和 $20.8 \mu \mathrm{mol} / \mathrm{L}$. 从红树植物木榄(B. gymnorrhiza $)$ 的内生真菌 P. thom $i$ 中分离得到了 1 个新的代 谢产物 4',5-dihydroxy-2,3-dimethoxy-4-(hydroxypropyl)biphenyl $(\mathbf{2 5 3})^{[40]}$, 且该化合物对人肿瘤细胞 A549, HepG2 和 HT29 有明显的生长抑制活性, 其 $\mathrm{IC}_{50}$ 值分别 为 $10.1 \pm 2.6,12.2 \pm 2.3$ 和 $8.9 \pm 1.9 \mu \mathrm{mol} / \mathrm{L}$. 从红树植物 白骨壤 (A. marina) 的内生真菌 P. brocae MA-192 的培养 液中分离鉴定出了 2 个新的开环的间苯二酚酸衍生 物 brocaketones C, D $(\mathbf{2 5 4}, \mathbf{2 5 5})^{[50]}$, 化合物 $\mathbf{2 5 4}$ 对 2,2联氮-二(3-乙基-苯并噻唑-6-磺酸)二铵盐(ABTS)自由 基表现出中等的抗氧化活性, 其 $\mathrm{IC}_{50}$ 值为 $14.4 \mu \mathrm{g} / \mathrm{mL}$. 从半红树植物黄槿(H. tiliaceus) 的内生真菌 P. commune $\mathrm{G} 2 \mathrm{M}$ 的培养物中分离得到了 1 个新的化合物 1-O-(2,4-dihydroxy-6-methylbenzoyl)-glycerol (256 $)^{[79]}$. 从红树植物木榄 (B. gymnorrhiza) 的内生真菌 Penicillium sp. GD6 的培养物中分离出了 1 个新的 sorbicillin 衍生物 2-deoxy-sohirnone C (257) ${ }^{[39]}$, 该化 合物对耐甲氧西林金黄色葡萄球菌(Methicillinresistant S. aureus) 显示出一定的抑制活性, 其 MIC 值 为 $80 \mu \mathrm{g} / \mathrm{mL}$. 从红树植物秋茄 $(K$. candel $)$ 的内生真菌 P. chermesinum $\mathrm{ZH} 4-\mathrm{E} 2$ 培养物中分离得到了 3 个新的 对联三苯类化合物 6'-O-desmethylterphenyllin (258), 3-hydroxy-6'-O-desmethylterphenyllin (259)和 3"-deoxy6'-O-desmethylcandidusin B (260) ${ }^{[22]}$, 这 3 个化合物均 对 $\alpha$-葡萄糖苷酶有较强的抑制作用, 其 $\mathrm{IC}_{50}$ 值分别为 $0.9,4.9$ 和 $2.5 \mu \mathrm{mol} / \mathrm{L}$. 从红树林根际海泥来源的真菌 P. javanicum HK1-22 中分离到了 2 个新的不饱和脂肪 酸 6R,8R-dihydroxy-9Z,12Z-octadecadienoic acid (261), methyl-6R,8R-dihydroxy-9Z,12Z-octadecadienoate (262) 和 1 个脂肪酸衍生物((4R,6R)-6-((1Z,4Z)-Deca-1,4-dien1-yl)-2,2-Dimethyl-1,3-dioxan-4-yl)pentanoic acid (263) ${ }^{[1]}$. 从红树植物木榄 (B. gymnorrhiza) 的内生真菌 Penicillium sp. GD6 培养物中分离鉴定出了 1 个新的甾醇类 化合物 11- $O$-acetyl-NGA0187 (264) ${ }^{[37]}$. 从红树植物白骨 壤(A. marina) 的内生真菌 P. brocae MA-192 提取物中分 离到了 2 个新化合物 brocaketones A, B $(\mathbf{2 6 5}, \mathbf{2 6 6})^{[49]}$, 这 两个化合物均对 $\mathrm{ABTS}$ 自由基显示活性，其 $\mathrm{IC}_{50}$ 值分别 为 8.7 和 $5.9 \mu \mathrm{g} / \mathrm{mL}$; 对 DPPH 自由基表现出中等的抗 
<smiles>CC(C)(C)C(O)COc1ccc(CCO)cc1</smiles>

247<smiles>Cc1cc(O)c(O)cc1-c1cc(O)cc(O)c1</smiles>

248<smiles>[R]c1ccc(Cc2c(C)cc(Oc3cc(C)cc(O)c3)cc2O)cc1O</smiles>

$249 \mathrm{R}=$

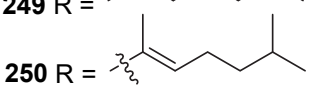

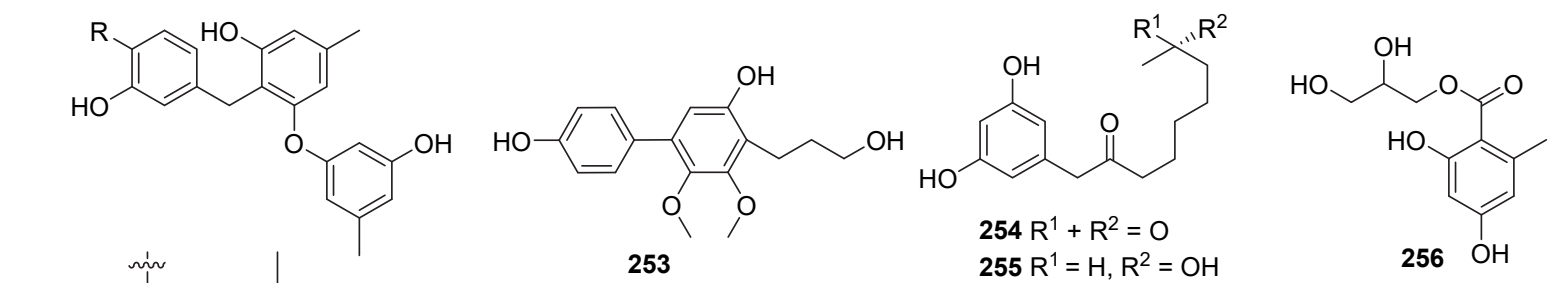

253<smiles>[R]c1cc(-c2c(O)cc(-c3ccc(O)cc3)c(OC)c2O)ccc1O</smiles>
$259 \mathrm{R}^{1}=\mathrm{OH}$<smiles>COc1c(-c2ccc(O)cc2)cc(O)c2c1oc1cc(O)c(O)cc12</smiles>

257<smiles>CCCCC/C=C/C/C=C/[C@H](O)C[C@@H](O)CCCCC(=O)O</smiles>

261<smiles>CCCCC/C=C\C/C=C\C(O)CC(O)CCCCC(=O)O[Na]</smiles>
262

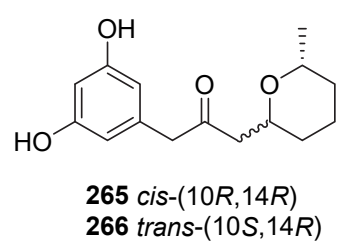

264<smiles>COc1c(/C=C/C(C)C)ccc(Oc2c(O)cc(C)cc2CO)c1C(=O)O</smiles>

267<smiles>COc1c([C@@H](O)C=C(C)C)ccc(Oc2c(O)cc(C)cc2CO)c1C(=O)O</smiles>

271<smiles>C=C(C)CCCCC(O)(O)c1ccc(C(=O)O)cc1O</smiles><smiles>COc1c(/C=C/C(C)C)ccc(Oc2c(O)cc(C)cc2COC(C)=O)c1C(=O)O</smiles>

268<smiles>COc1cc(C)cc(Oc2cc(C)cc(O)c2)c1</smiles>

269<smiles>C=C/C=C/CC(O)C/C=C\C=C/C(C)=O</smiles>

273<smiles>CC(=O)OC[C@@H](C)CCCCC(O)(O)c1ccc(C(=O)O)cc1O</smiles><smiles>CC(=O)OCc1cc(C)cc(O)c1Oc1ccc(CC=C(C)C)c(O)c1C(=O)O</smiles>

270<smiles>[Z20]c1cccc([C@@H]2O[C@H](C)[C@@H](C)c3c(O)cc(O)c(C)c32)c1</smiles>

274

图 8 红树来源青霉属真菌产生的其他类化合物 247 276

Figure 8 Other compounds $\mathbf{2 4 7} \sim \mathbf{2 7 6}$ produced by mangrove-derived Penicillium $\mathrm{sp}$. 
表 8 其他类新化合物 $\mathbf{2 4 7} \sim \mathbf{2 7 6}$ 的生物活性与产生菌的来源 ${ }^{a}$

Table 8 Biological activities of other new compounds $247 \sim 276$ and the resources of producing strains

\begin{tabular}{|c|c|c|c|c|}
\hline Compound & Source & Bioactivity (MIC, $\left.\mathrm{LD}_{50}, \mathrm{IC}_{50}\right)$ & Region & Year \\
\hline 247 & Leaves of $A$. marina & Tca8113: $2.0 \mu \mathrm{mol} / \mathrm{L}, \mathrm{MG}-63: 35 \mu \mathrm{mol} / \mathrm{L}$ & Fujain & $2014^{[48]}$ \\
\hline 248 & Branch of C. manghas & 1 & Hainan & $2015^{[60]}$ \\
\hline $249 \sim 252$ & E. agallocha & HL-60: 18.2/20.8 $\mu \mathrm{mol} / \mathrm{L}(\mathbf{2 5 0}, \mathbf{2 5 1})$ & Hainan & $2012^{[83]}$ \\
\hline 253 & Root of $B$. gymnorrhiza & A549/HepG2/HT29: $8.9 \sim 12.2 \mu \mathrm{mol} / \mathrm{L}$ & Guangxi & $2015^{[40]}$ \\
\hline $254 / 255$ & Laves of $A$. marina & ABTS: $14.4 \mu \mathrm{g} / \mathrm{mL}(\mathbf{2 5 4})$ & Hainan & $2015^{[50]}$ \\
\hline 256 & H. tiliaceus & 1 & Guangdong & $2010^{[79]}$ \\
\hline 257 & Stem of $B$. gymnorrhiza & Methicillin-resistant S. aureus: $80 \mu \mathrm{g} / \mathrm{mL}$ & Guangdong & $2018^{[39]}$ \\
\hline 258 & Stem of $K$. candel & $\alpha$-glucosidase: $0.9 \mu \mathrm{mol} / \mathrm{L}$ & Guangdong & $2011^{[22]}$ \\
\hline 259 & Stem of $K$. candel & $\alpha$-glucosidase: $4.9 \mu \mathrm{mol} / \mathrm{L}$ & Guangdong & $2011^{[22]}$ \\
\hline 260 & Stem of $K$. candel & $\alpha$-glucosidase: $2.5 \mu \mathrm{mol} / \mathrm{L}$ & Guangdong & $2011^{[22]}$ \\
\hline 261 & Rhizospheric soil of mangrove plant & / & Hainan & $2019^{[1]}$ \\
\hline 262 & Rhizospheric soil of mangrove plant & l & Hainan & $2019^{[1]}$ \\
\hline 263 & Rhizospheric soil of mangrove plant & / & Hainan & $2019^{[1]}$ \\
\hline 264 & Stem of $B$. gymnorrhiza & l & Guangdong & $2014^{[37]}$ \\
\hline 265,266 & Leaves of $A$. marina & $\begin{array}{l}\text { ABTS: } 8.7 / 5.9 \mu \mathrm{g} / \mathrm{mL} \\
\text { DPPH: } 18.7 / 16.3 \mu \mathrm{g} / \mathrm{mL}\end{array}$ & Hainan & $2015^{[50]}$ \\
\hline 267,268 & B. gymnorrhiza & A. salina: $14.2 / 39.2 \mu \mathrm{mol} / \mathrm{L}$ & Hainan & $2014^{[42]}$ \\
\hline 269 & Root of E. agallocha & 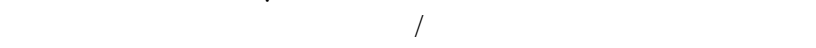 & Hainan & $2012^{[82]}$ \\
\hline 270 & Soil of $B$. gymnorrhiza & M. luteus/E. coli: $64 / 16 \mu \mathrm{g} / \mathrm{mL}$ & Hainan & $2012^{[41]}$ \\
\hline 271 & Soil of B. gymnorrhiza & 1 & Hainan & $2012^{[41]}$ \\
\hline 272 & Rhizospheric soil of mangrove plant & l & Fujian & $2020^{[16]}$ \\
\hline 273 & K. candel & AChE/ $\alpha$-glucosidase /Antioxidant: $>100 \mu \mathrm{mol} / \mathrm{L}$ & Hainan & $2020^{[27]}$ \\
\hline 274 & A. marina & 1 & Fujian & $2015^{[52]}$ \\
\hline 275,276 & Root of E. agallocha & HL-60: $15.7 \mu \mathrm{mol} / \mathrm{L}(\mathbf{2 7 5})$, A549/HL-60: 1.9, $5.4 \mu \mathrm{mol} / \mathrm{L}(\mathbf{2 7 6})$ & Hainan & $2010^{[82]}$ \\
\hline
\end{tabular}

${ }^{a} /$ : No activity.

氧化活性, 其 $\mathrm{IC}_{50}$ 值分别为 18.7 和 $16.3 \mu \mathrm{g} / \mathrm{mL}$. 从红树 植物木榄 (B. gymnorrhiza) 的内生真菌 Penicillium sp. MA-37 中分离出了 2 个新的二苯醚衍生物 penikellides A, B $(\mathbf{2 6 7}, 268)^{[42]}$, 化合物 267, 268 对卤虫(A. salina) 表 现出一定的致死活性，其 $\mathrm{LD}_{50}$ 值分别为 $14.2,39.2$ $\mu \mathrm{mol} / \mathrm{L}$. 从红树植物海漆 (E. agallocha) 的内生真菌 $P$. expansum 091006 培养物中分离出了 1 个新的二苯醚衍 生物 3-O-methyldiorcinol (269) ${ }^{[82]}$. 从红树植物木榄 $(B$. gymnorrhiza) 的内生真菌 Penicillium sp. MA-37 的代谢 产物中分离出了 2 个新的二苯醚衍生物 7-O-acetylsecopenicillide C (270)和 hydroxytenellic acid B (271) $)^{[41]}$, 其中化合物 271 对藤黄微球菌 (M. luteus)、大肠杆菌 ( $E$. coli) 显示出一定的抑制活性, 其 MIC 值分别为 64 和 16 $\mu \mathrm{g} / \mathrm{mL}$. 从红树植物秋茄 (K. candel) 的内生真菌 Penicillium sp. (SBE-8)的发酵提取物中分离到了 1 个新 的代谢产物 7-hydroxyjanthinone (272) ${ }^{[26]}$. 从红树植物 秋茄 $(K$. candel $)$ 的内生真菌 P. citrinum QJF-22 培养物 中分离得到 1 个新的化合物( $3 E, 5 Z, 8 S, 10 E$ )-8-hydroxytrideca-3,5,10,12-tetraen-2-one (273) ${ }^{[25]}$, 化合物 273 具有 较弱的抗氧化、乙酰胆碱酷酶 (AchE) 和 $\alpha$-葡萄糖苷酶活 性, 其 $\mathrm{IC}_{50}$ 值都大于 $100 \mu \mathrm{mol} / \mathrm{L}$. 从红树植物白骨壤 $(A$. marina) 的内生真菌 P. citrinum 中分离出了 1 个新化合物
(1S,3R,4S)-1-(40-hydroxyl-phenyl)3,4-dihydro-3,4,5-trimethyl-1H-2-benzopyran-6,8-diol (274) ${ }^{[52]}$. 从红树植物海漆 (E. agallocha) 的内生真菌 P. expansum 091006 的提取物 中分离出了 2 个新的化合物 expansols A, B $(\mathbf{2 7 5}, \mathbf{2 7 6})^{[82]}$, 其中化合物 275 对 HL-60 细胞具有中度的细胞毒性, 其 $\mathrm{IC}_{50}$ 值为 $15.7 \mu \mathrm{mol} / \mathrm{L}$, 化合物 276 对 A549 和 HL-60 细 胞具有明显的细胞毒活性, 其 $\mathrm{IC}_{50}$ 分别为 1.9 和 5.4 $\mu \mathrm{mol} / \mathrm{L}$.

\section{3 结论与展望}

通过查阅文献发现, 涉及到海洋来源的青霉属真菌 次级代谢产物的文献共有 700 余篇, 其中来源于红树及 其根际海泥的有 87 篇. 近十几年从红树来源青霉属真 菌中分离鉴定出 276 个新的结构多样的次级代谢产物, 包括生物碱类、肽类、萜类和聚酮类等(图 9A), 其中 19 个为具有新颖骨架的化合物; 相对于其他生境来源青霉 属真菌, 红树来源的青霉属真菌次级代谢产物多含有螺 环或桥环结构, 从中分离获得 23 个螺环类化合物(18, $21,22,33,43 \sim 45,105,106,49,213,243,244$ ) 和 30 个桥 环类化合物 $(4,7 \sim 11,19,20,28 \sim 30,34 \sim 41,43 \sim 51$, 67, 72). 同时由于红树植物生长于酸性或含盐等的生长 环境，从红树来源青霉属真菌中分离获得了 28 个不同 
于其他生境来源青霉属真菌的含硫二酮哌嗪类生物碱 化合物 $(\mathbf{1} \sim \mathbf{1 7}, \mathbf{1 9} \sim \mathbf{2 3}, \mathbf{2 8} \sim \mathbf{3 3})$ 以及 12 个 $(\mathbf{2 8}, \mathbf{3 1}, \mathbf{7 6}$,

$136,166,179,180,183,184,213,215$ 和 216)含有卤素的 化合物, 说明了生长环境对青霉属代谢产物次级代谢产 物生物合成途径产生影响. 通过生物活性篎选, 发现其 中 140 个化合物具有抗菌、酶抑制剂和抗肿瘤等生物活 性，占代谢产物总数的 $50.7 \%$ (图 9B), 48 个化合物具有 抗菌活性, 39 个化合物具有细胞毒活性, 26 个化合物个 具有酶抑制剂活性, 5 个化合物个具有多种生物活性.

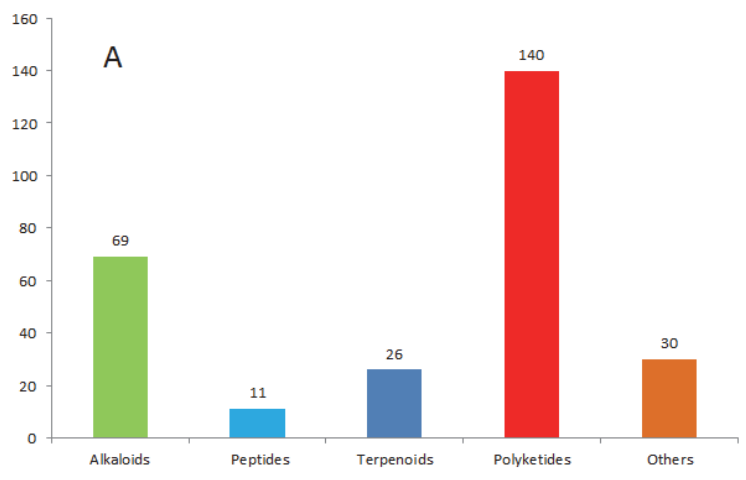

B

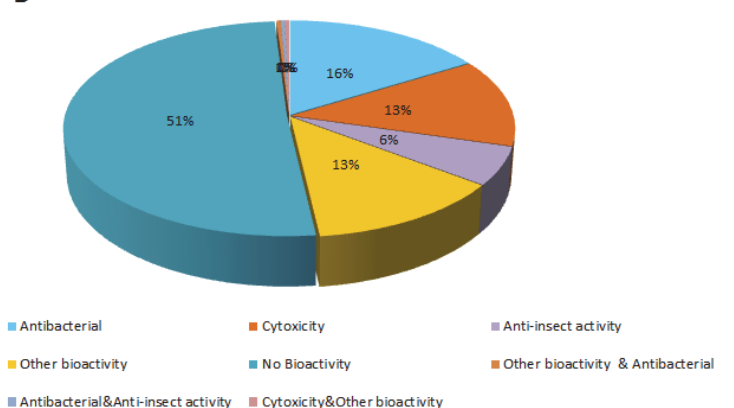

图 9 (A)报道的新天然产物的结构类型及(B)报道的新天然产 物的生物活性

Figure 9 (A) Ratio of new natural products based on structures and $(\mathrm{B})$ ratio of bioactive natural products

目前对红树来源青霉属真菌的研究主要集中于结 构新颖次级代谢产物的分离鉴定与生物活性初步篎选 评价, 仅有少数文献报道通过改变培养条件或组合生物 合成的方法对青霉属真菌次级代谢产物生物合成的关 键基因和关键酶进行研究. 基于以上研究结果, 在以后 红树来源青霉属真菌的研究过程中, 要注意加强青霉属 真菌化合物生物合成特定关键基因的寻找确定，对化合 物进行多种生物活性模型的篎选, 对活性化合物进行深 入的作用机制研究, 以确定疾病的靶标, 从而获得更多 药物先导化合物.

\section{References}

[1] Liang, Z. Y.; Shen, N. X.; Zheng, Y. Y.; Wu, J. T.; Li, M.; Fu, X. M.; Chen, M.; Wang, C. Y. Bioorg. Chem. 2019, 93, 103331.
[2] Chen, H. Q.; Qiu, L. M.; Wang, P.; Cai, C. H.; Wang, H.; Dai, H. F.; Mei, W. L. Photochem. Lett. 2019, 33, 36.

[3] Zheng, Y. Y.; Liang, Z. Y.; Shen, N. X.; Liu, W. L.; Zhou, X. J.; Fu, X. M.; Chen, M.; Wang, C. Y. Mar. Drugs 2019, 17, 3221.

[4] Chen, M.; Zheng, Y. Y.; Chen, Z. Q.; Shen, N. X.; Shen, L.; Zhang, F. M.; Zhou, X. J.; Wang, C. Y. J. Nat. Prod. 2019, 82, 368.

[5] Zheng, Y. Y.; Shen, N. X.; Liang, Z. Y.; Shen, L.; Chen, M.; Wang, C. Y. Nat. Prod. Res. 2020, 34, 378.

[6] Chen, M.; Shen, N. X.; Chen, Z. Q.; Zhang, F. M.; Chen, Y. J. Nat. Prod. 2017, 80, 1081.

[7] Zheng, Y. Y.; Chen, X; Chen, L, M.; Shen, L.; Fu, X. M.; Chen, Q. X.; Chen, M.; Wang, C. Y. J. Ocean Univ. China 2020, 19, 700.

[8] Tao, H. M.; Wei, X. Y.; Lin, X. P.; Zhou, X. F.; Dong, J. D.; Yang, B. Nat. Prod. Res. 2017, 31, 2218.

[9] Yang, B.; Dong, J. D.; Lin, X. P.; Zhou, X. F.; Zhang, Y. Y.; Liu, Y Y. Tetrahedron 2014, 70, 3859.

[10] Huang, J. X.; She, J. L.; Yang, X. L.; Liu, J.; Zhou, X. F.; Yang, B. Molecules 2019, 24, 16862.

[11] Yang, B.; Tao, H. M.; Lin, X. P.; Wang, J. F.; Liao, S. R.; Dong, J. D.; Zhou, X. F.; Liu, Y. H. Tetrahedron 2018, 74, 77.

[12] He, F.; Li, X. B.; Yu, J. H.; Zhang, X. Y.; Nong, X. H.; Chen, G. Y.; Zhu, K. K.; Wang, Y. Y.; Bao, J.; Zhang, H. Fitoterapia 2019, 136, 104177.

[13] Wu, C. M.; Zhao, Y.; Chen, R.; Liu, D.; Liu, M. Y.; Peter, P.; Guo, P.; Lin, W. H. RSC Adv. 2016, 6, 21969.

[14] Fan, Y. Q.; Wang, Y.; Liu, P. P.; Fu, P.; Zhu, T. H.; Wang, W.; Zhu, W. M. J. Nat. Prod. 2013, 76, 1328.

[15] Fan, Y. Q.; Zhu, G. L.; Wang, Y.; Zhu, X. C.; Gong, Q. H.; Jia, Q.; Fu, P.; Zhu, W. M. Chin. J. Org. Chem. 2018, 38, 2798 (in Chinese).

(樊亚琴, 朱国良，王义，朱晓翠，宫倩红，贾茜，付鹏，朱伟明， 有机化学, 2018, 38, 2798.)

[16] Fan, Y. Q.; Wang, Y.; Fu, P.; Arthit, C.; Pawinee, P.; Zhu, W. M. Org. Chem. Front. 2018, 5, 2835.

[17] Yang, S. Q.; Attila, M.; Li, X. M.; Liu, H.; Li, X.; Sandor, B. K.; Tibor, K.; Wang, B. G. Bioorg. Chem. 2021, 106, 104477.

[18] Liu, S. Z.; Tang, X. X.; He, F. M.; Jia, J. X.; Hu, H.; Xie, B. Y. Li, M. Y.; Qiu, Y. K. Nat. Prod. Res. 2020, doi: 10.1080/14786419. 2020.1837811

[19] Liu, S. Z.; He, F. M.; Bin, Y. L.; Qiu, Y. K.; Li, C. F.; Xie, B. Y.; Tang, X. X. Nat. Prod. Res. 2020, doi: 10.1080/14786419. 2020.1817015

[20] Cai, R. L.; Wu, Y. N.; Chen, S. H.; Cui, H.; Liu, Z. M.; Li, C. Y.; She, Z. G. J. Nat. Prod. 2018, 81, 1376.

[21] Huang, H. R.; Liu, T.; Wu, X. E.; Guo, J. X.; Lan, X.; Zhu, Q.; Zheng, X.; Zhang, K. Nat. Prod. Res. 2017, 31, 2593.

[22] Huang, H. B.; Feng, X. J.; Xiao, Z. E.; Liu, L.; Li, H. X.; Ma, L.; Lu, Y. J.; Ju, J. H.; She, Z. G.; Lin, Y. C. J. Nat. Prod. 2011, 74, 997.

[23] Huang, X. S.; Sun, X. F.; Ding, B.; Lin, M.; Lan, L.; Huang, H. R.; She, Z. G. Planta Med. 2013, 79, 1572 .

[24] Ding, B.; Wang, Z. Y.; Huang, X. S.; Liu, Y. Y.; Chen, W. R.; She, Z. G. Nat. Prod. Res. 2016, 30, 2805.

[25] Huang, X. S.; Yang, B.; Sun, X. F.; Xia, G. P.; Liu, Y. Y.; Lin, M.; She, Z. G. Helv. Chim. Acta 2014, 97, 664.

[26] Guo, Z. Y.; Cheng, F.; Zou, K.; Wang, J. Z.; She, Z. G.; Lin, Y. C. Nat. Prod. Commun. 2009, 4, 1481 .

[27] Yang, W. C.; Chen, Y.; Cai, R. L.; Zou, G.; Wang, B.; She, Z. G. Chem. Biodiversity 2020, 17, e2000192.

[28] Bai, M.; Zheng, C. J.; Huang, G. L.; Mei, R. Q.; Wang, B.; Luo, Y. P.; Zheng, C.; Niu, Z. G.; Chen, G. Y. J. Nat. Prod. 2019, 82, 1155.

[29] Bai, M.; Huang, G. L.; Mei, R. Q.; Wang, B.; Luo, Y. P.; Nong, X. H.; Chen, G. Y.; Zheng, C. J. Mar. Drugs 2019, 17, 4332.

[30] Huang, G. L.; Zhou, X. M.; Bai, M.; Liu, Y. X.; Zhao, Y. L.; Luo, Y. P.; Niu, Y. Y.; Zheng, C. J.; Chen, G. Y. Mar. Drugs 2016, 14, 1771.

[31] He, K. Y.; Zhang, C.; Duan, Y. R.; Huang, G. L.; Yang, C. Y.; Lu, X. R.; Zheng, C. J.; Chen, G. Y. J. Antibiot. 2017, 70, 823.

[32] Zheng, C. J.; Liao, H. X.; Mei, R. Q.; Huang, G. L.; Yang, L. J.; 
Zhou, X. M.; Shao, T. M.; Chen, G. Y.; Wang, C. Y. Nat. Prod. Res. 2019, 33, 1127.

[33] Zheng, C. J.; Huang, G. L.; Xu, Y.; Song, X. M.; Yao, J.; Liu, H.; Wang, R. P.; Sun, X. P. Nat. Prod. Res. 2016, 30, 821.

[34] Xu, R.; Li, X. M.; Wang, B. G. Phytochem. Lett. 2016, 17, 114.

[35] Li, H. L.; Xu, R.; Li, X. M.; Yang, S. Q.; Meng, L. H.; Wang, B. G. Org. Lett. 2018, 20, 1465.

[36] Zheng, C. J.; Huang, G. L.; Tang, X. Z.; Wang, D. E.; Gong, X. L.; Zhang, Q.; Song, X. P.; Chen, G. Y. Chin. J. Org. Chem. 2014, 34, 1172 (in Chineses). (郑彩娟, 黄国雷, 唐雄肇, 王德能, 龚小路, 张强, 宋小平, 陈 光英, 有机化学, 2014, 34, 1172.)

[37] Zhou, Z. F.; Yang, X. H.; Liu, H. L.; Gu, Y. C.; Ye, B. P.; Guo, Y. W. Helv. Chim. Acta 2014, 97, 1564.

[38] Zhou, Z. F.; Kurtan, T. B.; Yang, X. H.; Geng, M. Y.; Ye, B. P.; Guo, Y. W. Org. Lett. 2014, 16, 1390.

[39] Jiang, C. S.; Zhou, Z. F.; Yang, X. H.; Lan, L. F.; Gu, Y. C.; Ye, B. P.; Guo, Y. W. Chin. J. Nat. Med. 2018, 16, 358.

[40] Chen, G.; Zhu, Y.; Wang, H. Z.; Wang, S. J.; Zhang, R. Q. J. Asian. Nat. Prod. Res. 2007, 9, 159.

[41] Zhang, Y.; Li, X. M.; Shang, Z.; Li, C. S.; Ji, N. Y.; Wang, B. G. J. Nat. Prod. 2012, 75, 1888

[42] Luo, H.; Li, X. M.; Li, C. S.; Wang, B. G. Phytochem. Lett. 2014, 9, 22.

[43] Meng, L. H.; Li, X. M.; Liu, Y.; Wang, B. G. Chin. Chem. Lett. $\mathbf{2 0 1 5}, 26,610$

[44] Meng, L. H.; Zhang, P.; Li, X. M.; Wang, B. G. Mar. Drugs 2015, $13,276$.

[45] Meng, L. H.; Li, X. M.; Lv, C. T.; Huang, C. G.; Wang, B. G. J. Nat. Prod. 2014, 77, 1921.

[46] Meng, L. H.; Wang, C. Y.; Li, X. M.; Hu, X. Y.; Wang, B. G. Org. Lett. 2016, 18, 5304.

[47] Meng, L. H.; Li, X. M.; Liu, Y.; Xu, G. M.; Wang, B. G. RSC. Adv. 2017, 7, 55026.

[48] Zheng, C.; Chen, Y.; Jiang, L. L.; Shi, X. M. Phytochem. Lett. 2014, $10,272$.

[49] Zhang, P.; Meng, L. H.; Mandi, A.; Kurtan, T.; Li, X. M.; Liu, Y.; Li, X.; Li, C. S.; Wang, B. G. Eur. J. Org. Chem. 2014, 2014, 4029.

[50] Zhang, P.; Meng, L. H.; Li, X. M.; Wang, B. G. RSC Adv. 2015, 5, 39870.

[51] Huang, Z. J.; Yang, J. X.; Cai, X. L.; She, Z. G.; Lin, Y. C. Nat. Prod. Res. 2012, 26, 1291.

[52] Liu, J.; X. M.; Zhu, M. Y.; Feng, Y. Nat. Prod. Commun. 2015, 10, 1203.

[53] Guo, W. Q.; Li, D.; Peng, J. X.; Zhu, T. J.; Gu, Q. Q.; Li, D. H. J. Nat. Prod. 2015, 78, 306.

[54] Li, H. X.; Jiang, J. Y.; Liu, Z. M.; Lin, S. E.; Xia, G. P.; Xia, X. K.; Ding, B.; He, L.; Lu, Y. J.; She, Z. G. J. Nat. Prod. 2014, 77, 800.

[55] Shao, C. L.; Wang, C. Y.; Wei, M. Y.; Gu, Y. C.; Xia, X. K.; She, Z. G.; Lin, Y. C. Magn. Reson. Chem. 2008, 46, 1066.

[56] Li, J.; Zhong, Y. S.; Yuan, J.; Zhu, X.; Lu, Y. J.; Lin, Y. C.; Lan, L. Nat. Prod. Commun. 2015, 10, 1549.

[57] Liu, J. F.; Chen, W. J.; Xin, B. R.; Lu, J. Nat. Prod. Commun. 2014, 9, 799.

[58] Zhang, P.; Li, X. M.; Liu, H.; Li, X.; Wang, B. G. Phytochem. Lett. 2015, 13, 160

[59] Guo, W. Q.; Kong, X. L.; Zhu, T. J.; Gu, Q. Q.; Li, D. H. Arch. Pharm. Res. 2015, 38, 1449.

[60] Liu, Y. Y.; Yang, Q.; Xia, G. P.; Huang, H. B.; Li, H. X.; Li, M.; Lu, Y. J.; He, L.; Xia, X. K.; She, Z. G. J. Nat. Prod. 2015, 78, 1816.

[61] Liu, Y. Y.; Xia, G. P.; Li, H. X.; Lin, M.; Ding, B.; Lu, Y. J.; He, L.; Xia, X. K.; She, Z. G. Planta Med. 2014, 80, 912.

[62] Lin, Z. J.; Zhu, T. J.; Fang, Y. C.; Gu, Q. Q.; Zhu, W. M. Phytochemistry 2008, 69, 1273.

[63] Xu, M. J.; Gessner, G. D.; Groth, I.; Lange, C.; Christner, A.; Bruhn, T.; Deng, Z. W.; Li, X.; Lin, W. H. Tetrahedron 2007, 63, 435.

[64] Zhu, M. L.; Yang, Z.; Feng, H. M.; Gan, Q.; Che, Q.; Zhu, T. J.; Gu,
Q. Q.; Han, B. N.; Li, D. H. RSC Adv. 2017, 7, 48019

[65] Zhu, M. L.; Zhang, X. M.; Feng, H. M.; Dai, J. J.; Li, J.; Che, Q.; Gu, Q. Q.; Zhu, T. J.; Li, D. H. J. Nat. Prod. 2017, 80, 71.

[66] Yang, Z.; Zhu, M. L.; Li, D. H.; Zeng, R.; Han, B. N. Bioorg. Med. Chem. Lett. 2017, 25, 6614.

[67] Zhu, M. L.; Yang, Z.; Wang, H, T.; Gan, Q.; Zhang, G. J.; Che, Q.; Zhu, T. J.; Gu, Q. Q.; Han, B. N.; Li, D. H. J. Nat. Prod. 2020, 83, 2647.

[68] Qiu, L. M.; Wang, P.; Liao, G.; Zeng, Y. B.; Cai, C. H.; Kong, F. D.; Guo, Z. K.; Peter, P.; Dai, H. F.; Mei, W. L. Mar. Drugs 2018, 16, 1082.

[69] Bai, M.; Zheng, C. J.; Nong, X. H.; Zhou, X. M.; Luo, Y. P.; Chen, G. Y. Mar. Drugs 2019, 17, 6922.

[70] Meng, L. H.; Li, X. M.; Lv, C. T.; Li, C. S.; Xu, G. M.; Huang, C. G.; Wang, B. G. J. Nat. Prod. 2013, 76, 2145.

[71] Meng, L. H.; Li, X. M.; Liu, Y.; Wang, B. G. Org. Lett. 2014, 16 , 6052.

[72] Meng, L. H.; Li, X. M.; Li, H. L.; Wang, B. G. Mar. Drugs 2020 , $18,3392$.

[73] Zhu, X. W.; Wu, Z. H.; Liang, F. Y.; Gan, S. X.; Huang, Q.; Ding, W. J.; Li, C. Y. Chem. Nat. Compd. 2018, 54, 520.

[74] Zhu, X. W.; Zhou, D. L.; Liang, F. Y.; Wu, Z. H.; She, Z. G.; Li, C. Y. Fitoterapia 2017, 123, 23.

[75] Huang, S.; Chen, H. Y.; Li, W. S.; Zhu, X. W.; Ding, W. J.; Li, C. Y. Mar. Drugs 2016, 14, 1721.

[76] Cao, J.; Li, X. M.; Li, X.; Li, H. L.; Meng, L. H.; Wang, B. G. Phytochem. Lett. 2019, 32, 1

[77] Liu, H. J.; Chen, S. H.; Liu, W. Y.; Liu, Y. Y.; Huang, X. S.; She, Z. G. Mar. Drugs 2016, 14, 2171.

[78] Ma, Y. H.; Li, J.; Huang, M. X.; Liu, L.; Wang, J.; Lin, Y. C. Mar. Drugs 2015, 13, 6306.

[79] Yan, H. J.; Gao, S. S.; Li, C. S.; Li, X. M.; Wang, B. G. Molecules 2010, 15, 3270.

[80] Darsih, C.; Prachyawarakorn, V.; Wiyakrutta, S.; Mahidol, C.; Ruchirawat, S.; Kittakoop, P. RSC Adv. 2015, 5, 70595.

[81] Qiu, P.; Cai, R. L.; Li, L.; She, Z. G. Chin. J. Nat. Med. 2020, 18, 256.

[82] Lu, Z. Y.; Zhu, H. J.; Fu, P.; Wang, Y.; Zhang, Z. H.; Lin, H. P.; Liu, P. P.; Zhuang, Y. B.; Hong, K.; Zhu, W. M. J. Nat. Prod. 2010, 73, 911.

[83] Wang, J. F.; Lu, Z. Y.; Liu, P. P.; Wang, Y.; Li, J.; Hong, K.; Zhu, W. M. Planta Med. 2012, 78, 1861.

[84] Shen, Y.; Huang, J. G.; Cai, L. H.; Wang, X. X.; Yang, S. T.; Huang, X. S.; Deng, M. C.; Duan, D. Guangdong Chem. Ind. 2020, 47, 85.

[85] Han, Z.; Mei, W. L.; Zhao, Y. X.; Deng, Y. Y.; Dai, H. F. Chem. Nat. Compd. 2009, 45, 805.

[86] Li, J.; Yang, X.; Lin, Y. Y.; Yuan, J.; Lu, Y. J.; Zhu, X.; Li, J.; Li, M. F.; Lin, Y. C.; He, J. G. Fitoterapia 2014, 97, 241.

[87] Zhang, Z. Z.; He, X. Q.; Zhang, G. J.; Che, Q.; Zhu, T. J.; Gu, Q. Q.; Li, D. H. J. Nat. Prod. 2017, 80, 3167.

[88] Wang, J. M.; Ding, G. Z.; Fang, L.; Dai, J. G.; Yu, S. S.; Wang, Y. H.; Chen, X. G.; Ma, S. G.; Qu, J.; Xu, S. J. Nat. Prod. 2010, 73, 1240.

[89] Guo, H. J.; Sun, B. D.; Gao, H.; Chen, X. L.; Liu, S. C.; Yao, X. S.; Liu, X. Z.; Che, Y. S. J. Nat. Prod. 2009, 72, 2115.

[90] Chi, L. P.; Li, X. M.; Li, X.; Wang, B. G. Chem. Biodiversity 2020, $17, \mathrm{e} 2000320$

[91] Harwoko, H.; Lee, J.; Hartmann, R.; Mandi, A.; Kurtan, T.; Mueller, W. E. G.; Feldbruegge, M.; Kalscheuer, R.; Ancheeva, E.; Daletos, G. Fitoterapia 2020, 146, 104698.

[92] Kong, F. D.; Wang, Y.; Liu, P. P.; Dong, T. H.; Zhu, W. M. J. Nat Prod. 2014, 77, 132.

[93] Zhou, L. M.; Kong, F. D.; Fan, P.; Ma, Q. Y.; Xie, Q. Y.; Li, J. H.; Zheng, H. Z.; Zheng, Z. H.; Yuan, J. Z.; Dai, H. F. J. Nat. Prod. 2019, 82, 2638.

[94] Hu, X. Y.; Meng, L. H.; Li, X.; Yang, S. Q.; Li, X. M.; Wang, B. G. Mar. Drugs 2017, 15, 13701.

[95] Gao, N.; Shang, Z. C.; Yu, P.; Luo, J.; Jian, K. L.; Kong, L. Y.; 
Yang, M. H. Chin. Chem. Lett. 2017, 28, 1194.

[96] Ariantari, N. P.; Ancheeva, E.; Wang, C. Y.; Mandi, A.; Knedel, T. O.; Kurtan, T.; Chaidir, C.; Mueller, W. E. G.; Kassack, M. U.; Janiak, C. J. Nat. Prod. 2019, 82, 1412.

[97] Zhao, J. C.; Wang, Y. L.; Zhang, T. Y.; Chen, Z. J.; Yang, T. M.; Wu, Y. Y.; Sun, C. P.; Ma, X. C.; Zhang, Y. X. Phytochemistry 2018, $148,21$.

[98] Gao, S. S.; Li, X. M.; Williams, K.; Proksch, P.; Ji, N. Y.; Wang, B. G. J. Nat. Prod. 2016, 79, 2066.

[99] Lo, H. C.; Entwistle, R.; Guo, C. J.; Ahuja, M.; Szewczyk, E.; Hung, J. H.; Chiang, Y. M.; Oakley, B. R.; Wang, C. C. C. J. Am. Chem. Soc. 2012, 134, 4709

[100] Arunpanichlert, J.; Rukachaisirikul, V.; Phongpaichit, S.; Supaphon, O.; Sakayaroj, J. Tetrahedron 2015, 71, 882 .
[101] Liu, Z. M.; Liu, H. J.; Chen, Y.; She, Z. G. Nat. Prod. Res. 2018 $32,2652$.

[102] Wen, H. L.; Yang, X. L.; Liu, Q.; Li, S. J.; Li, Q.; Zang, Y.; Chen, C. M.; Wang, J. P.; Zhu, H. C.; Zhang, Y. H. J. Nat. Prod. 2020, 83, 99.

[103] Jia, Q.; Du, Y. Q.; Wang, C.; Wang, Y.; Zhu, T. H.; Zhu, W. M. Mar Drugs 2019, 17, 2602.

[104] Cao, F.; Meng, Z. H.; Mu, X.; Yue, Y. F.; Zhu, H. J. J. Nat. Prod. 2019, 82,386

[105] Cao, F.; Yang, J. K.; Liu, Y. F.; Zhu, H. J.; Wang, C. Y. Nat. Prod. Res. 2016, 30, 2448

[106] Quang, D. N.; Hashimoto, T.; Nomura, Y.; Wollweber, H.; Hellwig, V.; Fournier, J.; Stadler, M.; Asakawa, Y. Phytochemistry 2005, 66 , 797.

(Zhao, C.) 